\title{
Chelate Palladium(II) Complexes with Saturated N-Phosphanyl-N- Heterocyclic Carbene (NHCP) Ligands: Synthesis and Catalysis
}

Anatoliy Marchenko, ${ }^{[\mathrm{a}]}$ Georgyi Koidan, ${ }^{[\mathrm{a}]}$ Anastasiia N. Hurieva, ${ }^{[\mathrm{a}]}$ Yurii Vlasenko, Aleksandr Kostyuk, * ${ }^{[a]}$ Andrea Biffis ${ }^{*[b]}$

${ }^{a}$ Institute of Organic Chemistry National Academy of Sciences of Ukraine, Murmanska 5, Kyiv-94, 02660, Ukraine. Homepage: www.ioch.kiev.ua

${ }^{\text {b}}$ Dipartimento di Scienze Chimiche, Università di Padova, via Marzolo 1, 35131 Padova, Italy.

\section{Supporting Information}

Crystallographic parameters of the compounds $\mathbf{3 b}$ and $\mathbf{9 a - 9 c}$; copies of spectra of new compounds 
Table S1. Main crystallographic parameters of the compounds $\mathbf{3 b}$ and $9 \mathbf{9 - 9}$.

\begin{tabular}{|c|c|c|c|c|}
\hline Compound & $\mathbf{3 b}$ & $9 a$ & 9b & $9 \mathrm{c}$ \\
\hline Cell Parameters & & & & \\
\hline$a[\AA]$ & $10.1402(3)$ & $9.4381(2)$ & $7.8616(3)$ & $7.5755(2)$ \\
\hline$b[\AA]$ & $14.3567(4)$ & $12.831(2)$ & $28.4967(10)$ & $29.6946(7)$ \\
\hline$c[\AA]$ & $14.9971(4)$ & $17.115(3)$ & $12.2214(4)$ & $12.1841(3)$ \\
\hline$\alpha\left[^{\circ}\right]$ & $93.788(1)$ & 90 & 90 & 90 \\
\hline$\beta\left[^{\circ}\right]$ & $100.686(1)$ & 90 & $103.744(2)$ & $101.2660(10)$ \\
\hline $\left.\mathcal{X}^{\circ}\right]$ & $90.570(1)$ & 90 & 90 & 90 \\
\hline $\mathrm{V}\left[\AA^{3}\right]$ & $2140.2(1)$ & $2072.5(6)$ & $2659.56(16)$ & $2688.02(12)$ \\
\hline $\mathrm{Z}$ & 4 & 4 & 2 & 4 \\
\hline $\mathrm{D}\left[\mathrm{g}^{\prime} \mathrm{cm}^{-3}\right]$ & 1.075 & 1.544 & 1.407 & 1.453 \\
\hline Crystal system & Triclinic & Orthorhombic & Monoclinic & Monoclinic \\
\hline Space group & $P-1$ & $P 2_{1} 2_{1} 2_{1}$ & $\mathrm{P} 2{ }_{1} / \mathrm{n}$ & $\mathrm{P} 2_{1} / \mathrm{n}$ \\
\hline$\mu\left[\mathrm{cm}^{-1}\right]$ & 0.133 & 1.233 & 0.973 & 1.043 \\
\hline $\mathrm{F}(000)$ & 760 & 984 & 1162 & 1216 \\
\hline \multirow[t]{3}{*}{ Indexes } & $12 \geq h \geq-13$ & $6 \geq h \geq-11$ & $9 \geq h \geq-9$ & $9 \geq h \geq-9$ \\
\hline & $19 \geq k \geq-19$ & $15 \geq k \geq-16$ & $33 \geq k \geq-35$ & $36 \geq k \geq-37$ \\
\hline & $20 \geq l \geq-20$ & $20 \geq l \geq-19$ & $14 \geq l \geq-15$ & $15 \geq l \geq-15$ \\
\hline$\theta_{\max }\left[{ }^{\circ}\right]$ & 29.3 & 26.5 & 26.4 & 26.7 \\
\hline \multicolumn{5}{|l|}{ No. of reflections: } \\
\hline collected & 34078 & 7586 & 24815 & 24771 \\
\hline independent & 11102 & 3839 & 5439 & 5662 \\
\hline in refinement & & & & \\
\hline$(I \geq 3 \sigma(I))$ & 6730 & 3210 & 4062 & 4566 \\
\hline$R$ (int) & 0.053 & 0.042 & 0.058 & 0.044 \\
\hline $\begin{array}{c}\text { No. of refined } \\
\text { parameters }\end{array}$ & 433 & 217 & 289 & 271 \\
\hline Obsd./var. & 15.5 & 14.7 & 14.1 & 16.8 \\
\hline \multicolumn{5}{|l|}{ Final $R$ indices } \\
\hline$R_{1}$ & 0.048 & 0.046 & 0.035 & 0.038 \\
\hline$R_{\mathrm{w}}$ & 0.097 & 0.050 & 0.032 & 0.034 \\
\hline GOF & 0.9092 & 1.072 & 1.094 & 1.108 \\
\hline
\end{tabular}




\begin{tabular}{|c|c|c|c|c|}
\hline Weighting & 11.9 & 4.02 & 1.21 & 1.13 \\
coefficients & 14.7 & -2.46 & -1.14 & -1.07 \\
& 5.91 & 3.23 & 0.607 & 0.622 \\
& & & -0.276 & -0.346 \\
\hline $\begin{array}{c}\text { Largest peak/hole } \\
{\left[\mathrm{e} \cdot \mathrm{cm}^{-3}\right]}\end{array}$ & $0.45 /-0.30$ & $1.26 /-0.64$ & $0.50 /-0.56$ & $2.16 /-0.78$ \\
\hline $\begin{array}{c}\text { CCDC deposition } \\
\text { number }\end{array}$ & 1437123 & 1437124 & 1437122 & 1437233 \\
\hline
\end{tabular}


Figure S1. ${ }^{1} \mathrm{H}$ NMR spectrum of $\mathbf{2 a}$

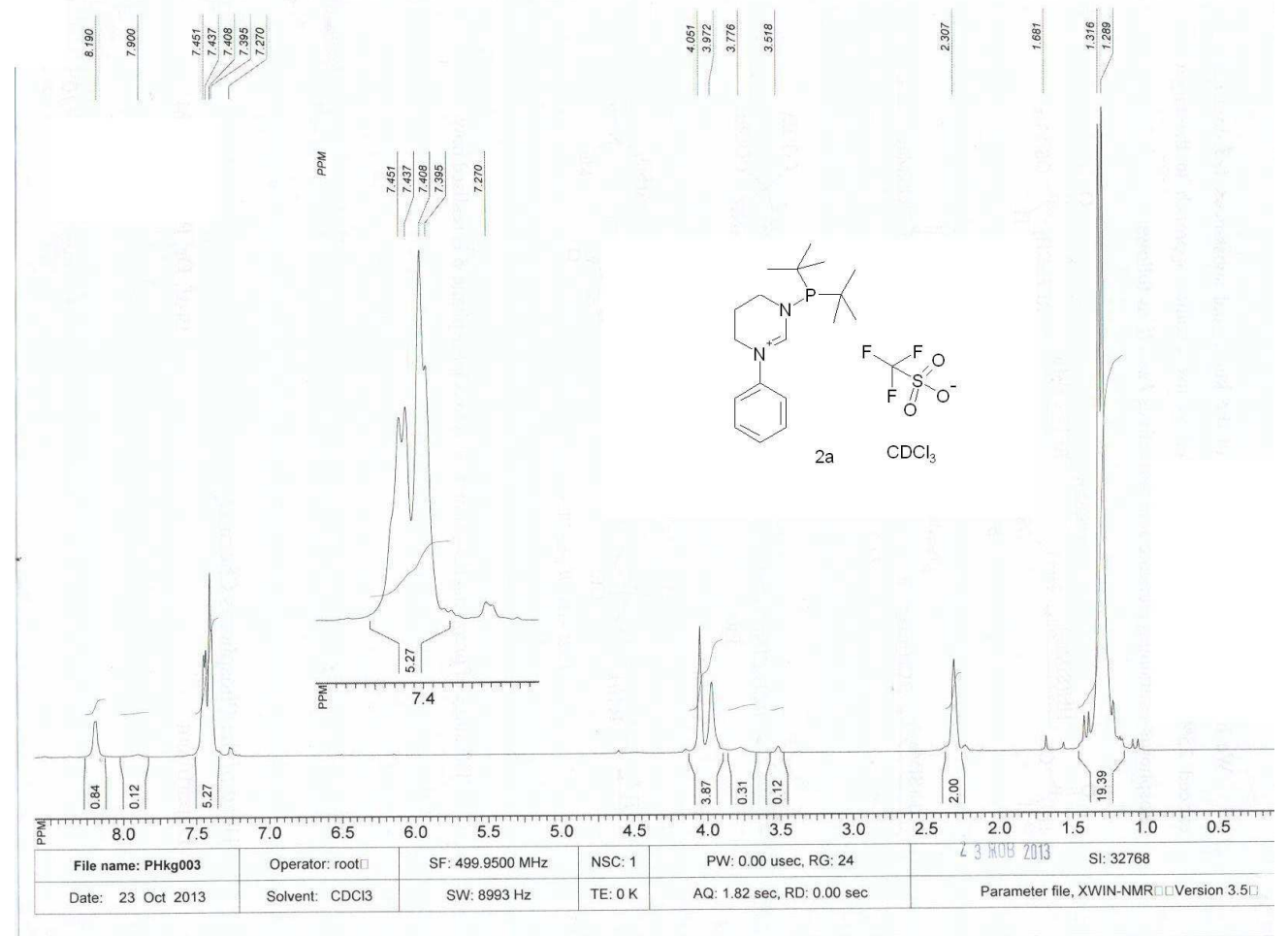

Figure S2. ${ }^{13} \mathrm{C}$ NMR spectrum of $\mathbf{2 a}$

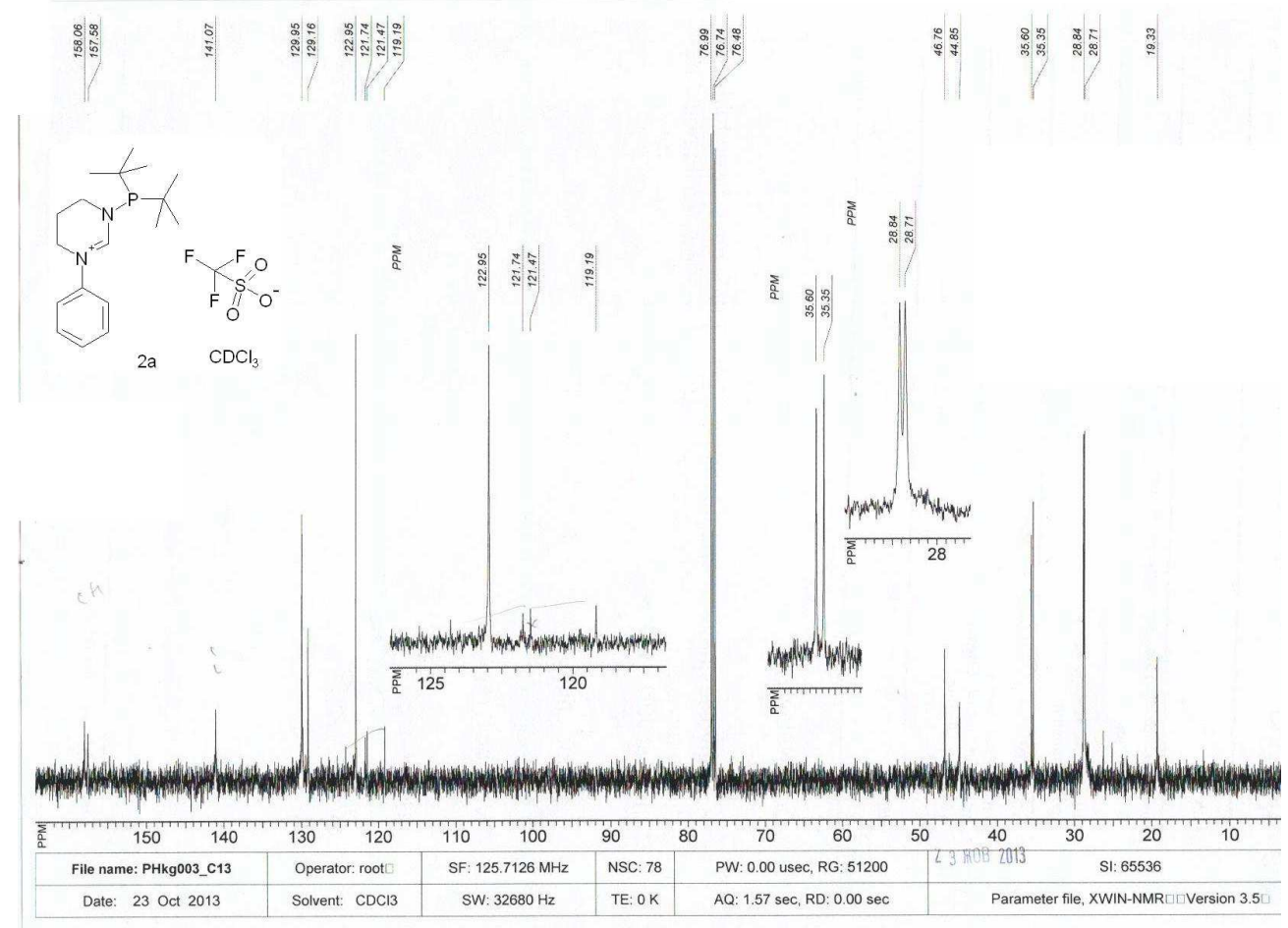


Figure $\mathrm{S} 3 .{ }^{31} \mathrm{P}$ NMR spectrum of $\mathbf{2 a}$

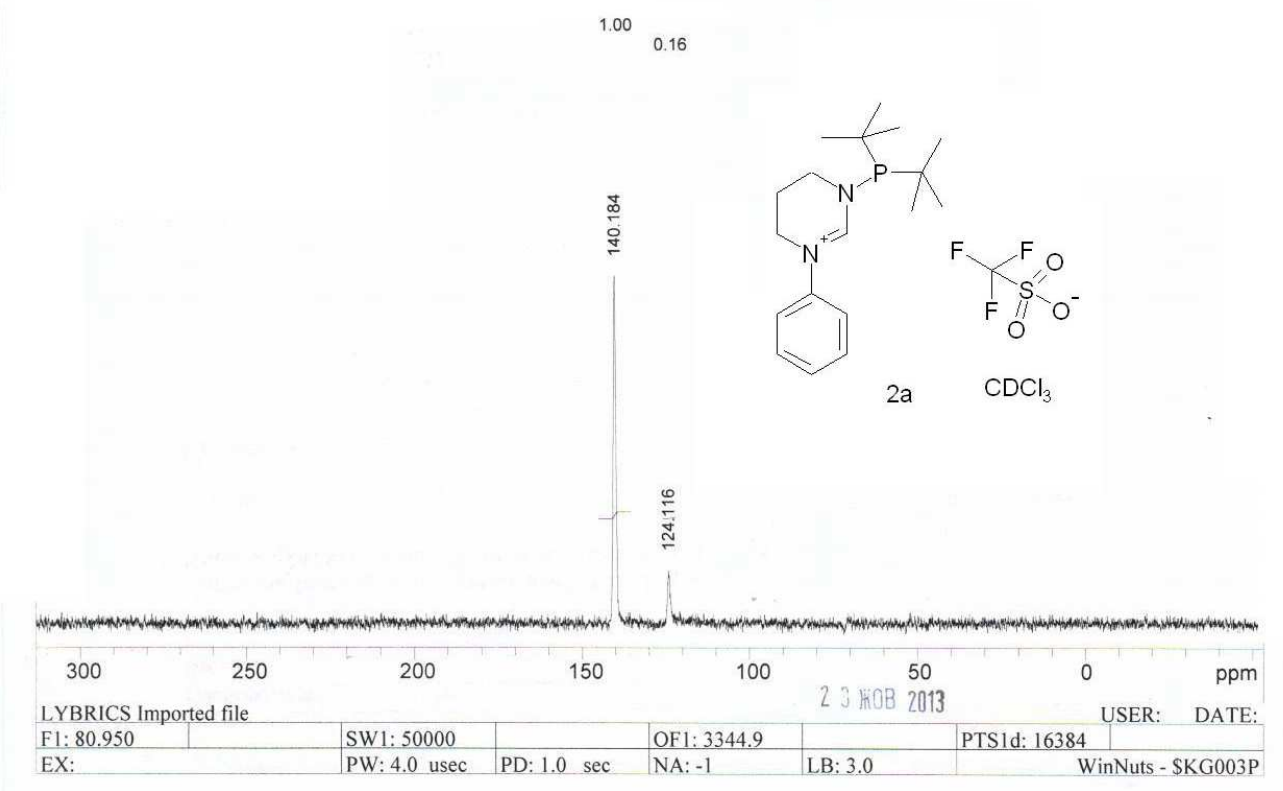

Figure S4. ${ }^{1} \mathrm{H}$ NMR spectrum of $\mathbf{2 b}$

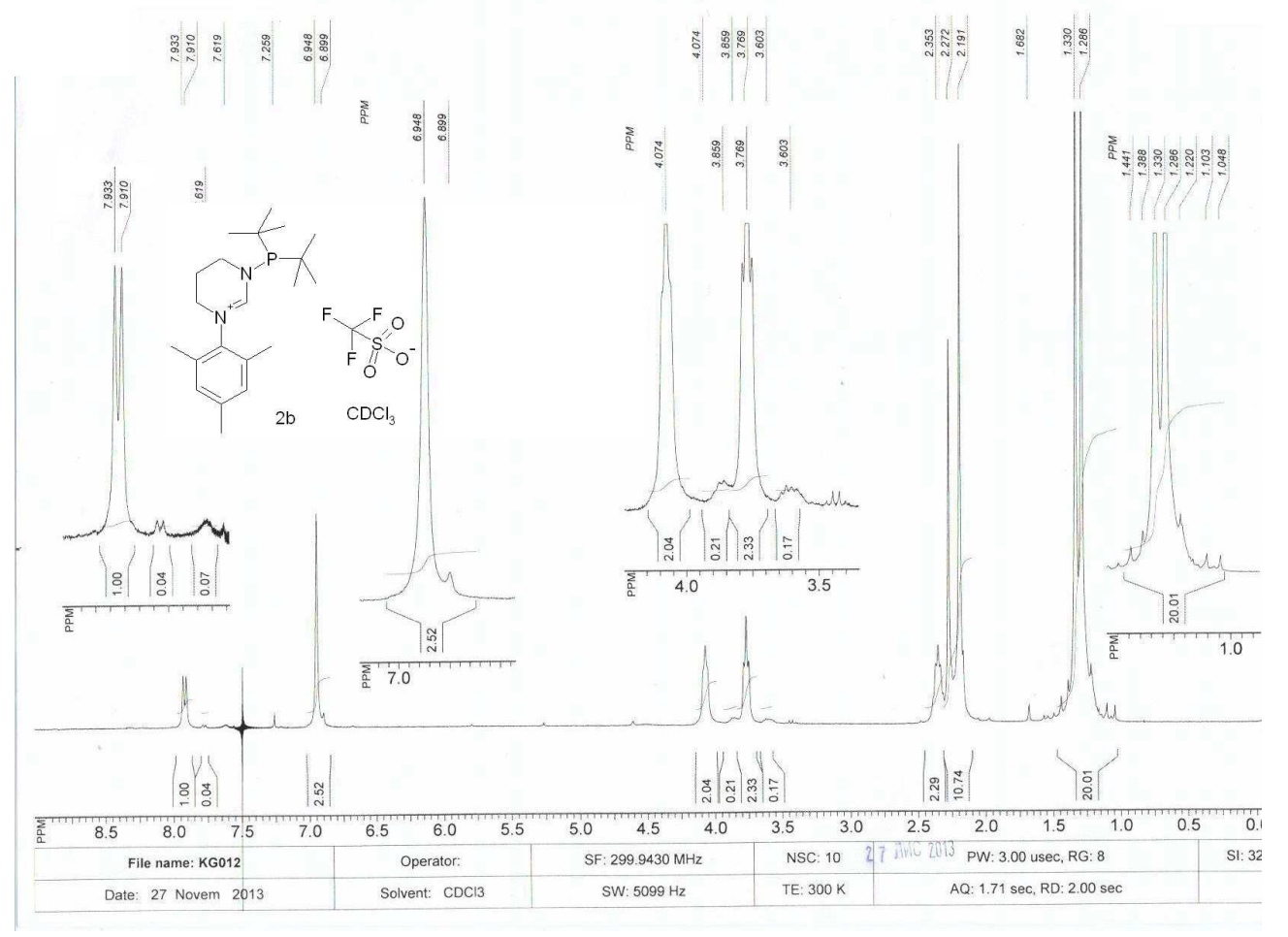


Figure S5. ${ }^{13} \mathrm{C}$ NMR spectrum of $\mathbf{2 b}$

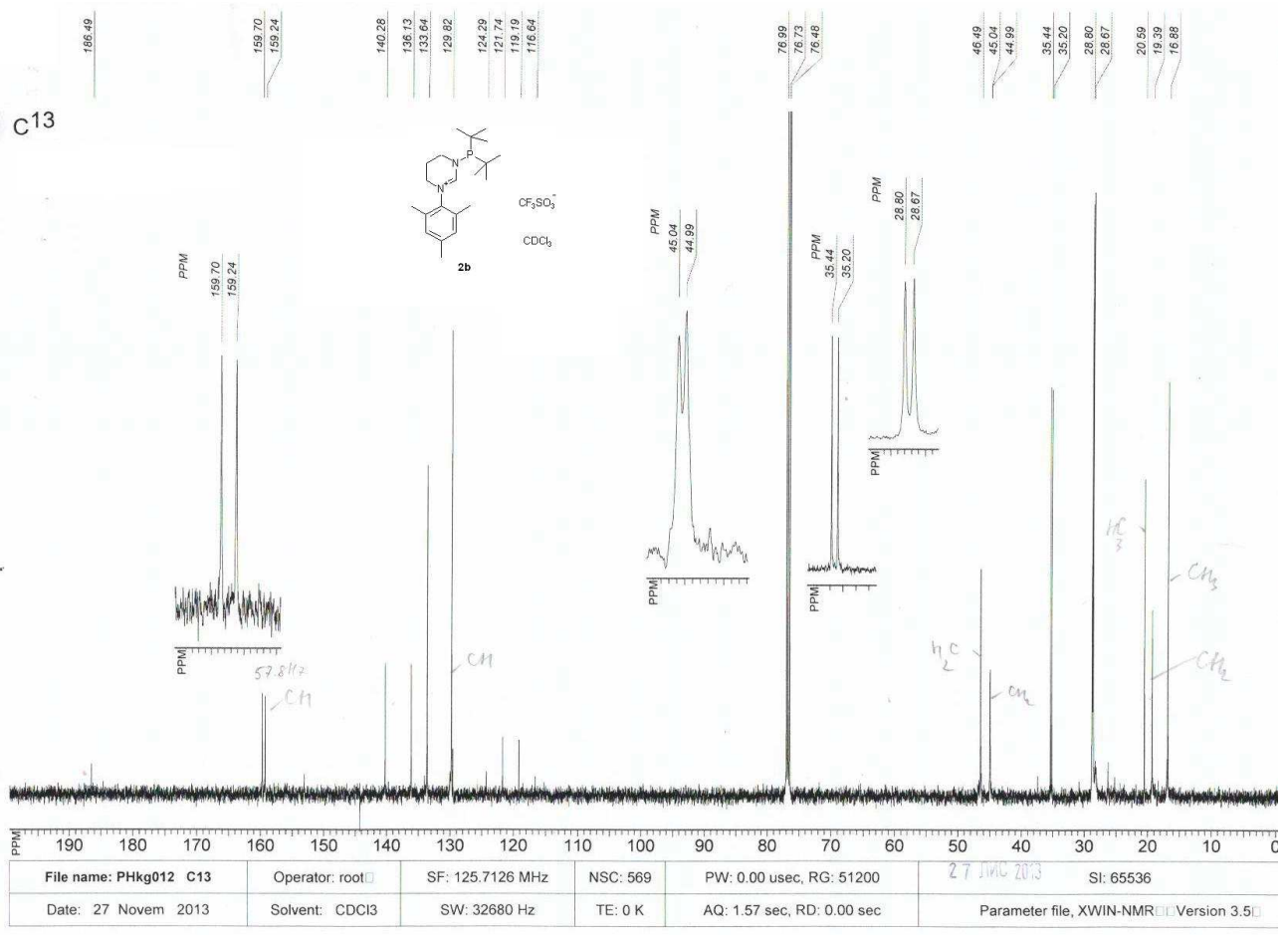

Figure S6. ${ }^{31} \mathrm{P}$ NMR spectrum of $\mathbf{2 b}$

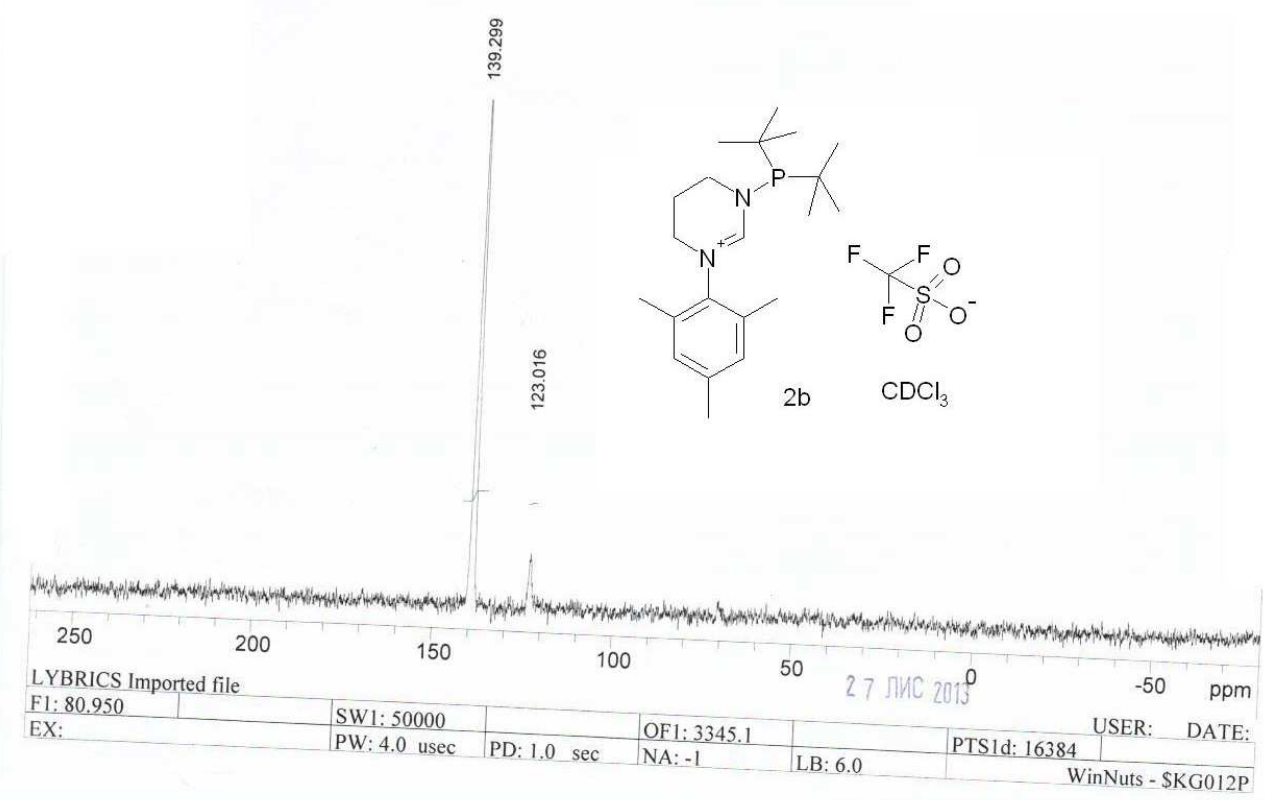


Figure S7. ${ }^{1} \mathrm{H}$ NMR spectrum of $\mathbf{3 a}$

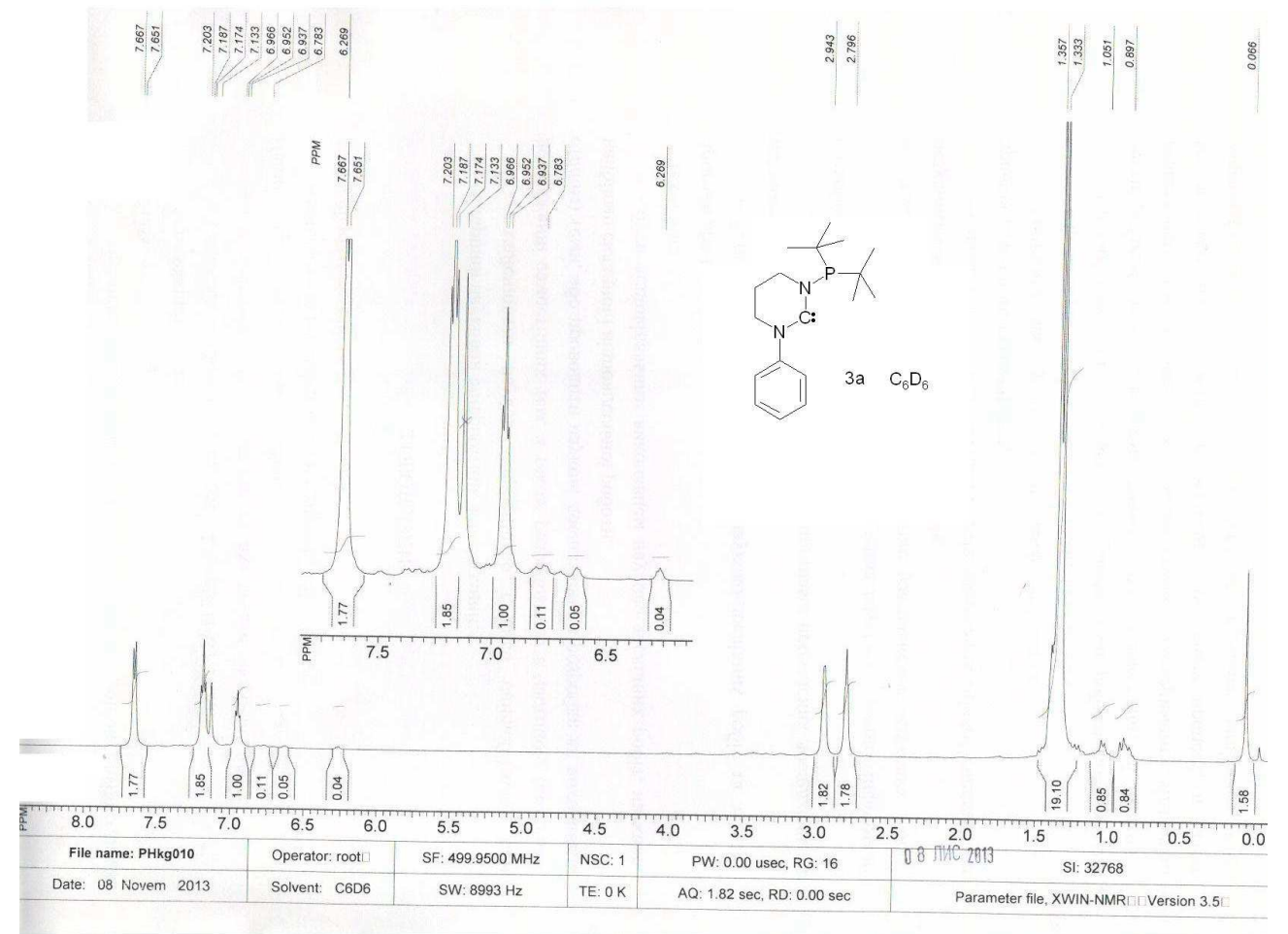

Figure S8. ${ }^{13} \mathrm{C}$ NMR spectrum of $\mathbf{3 a}$

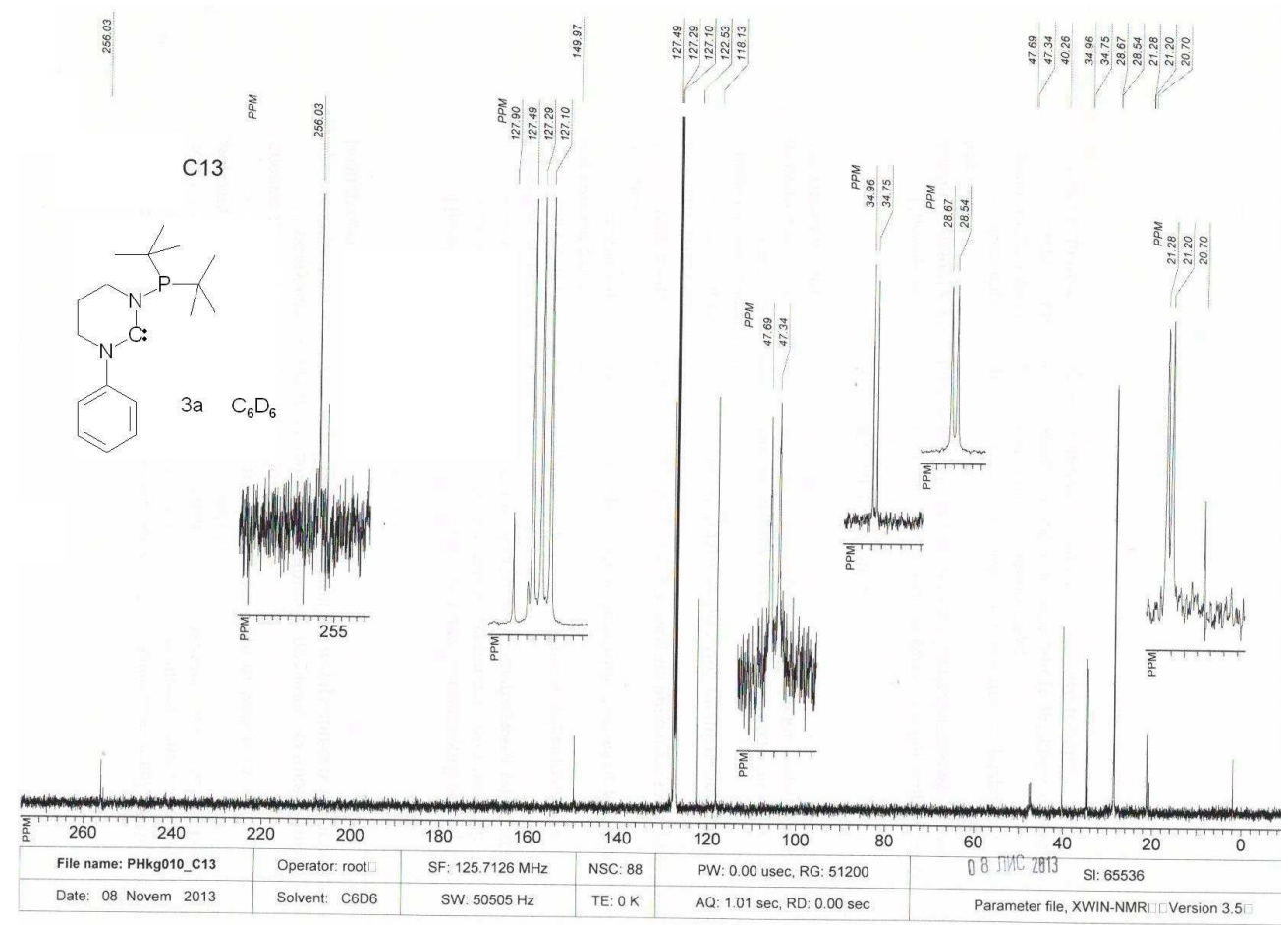


Figure S9. ${ }^{1} \mathrm{H}$ NMR spectrum of $\mathbf{3 b}$

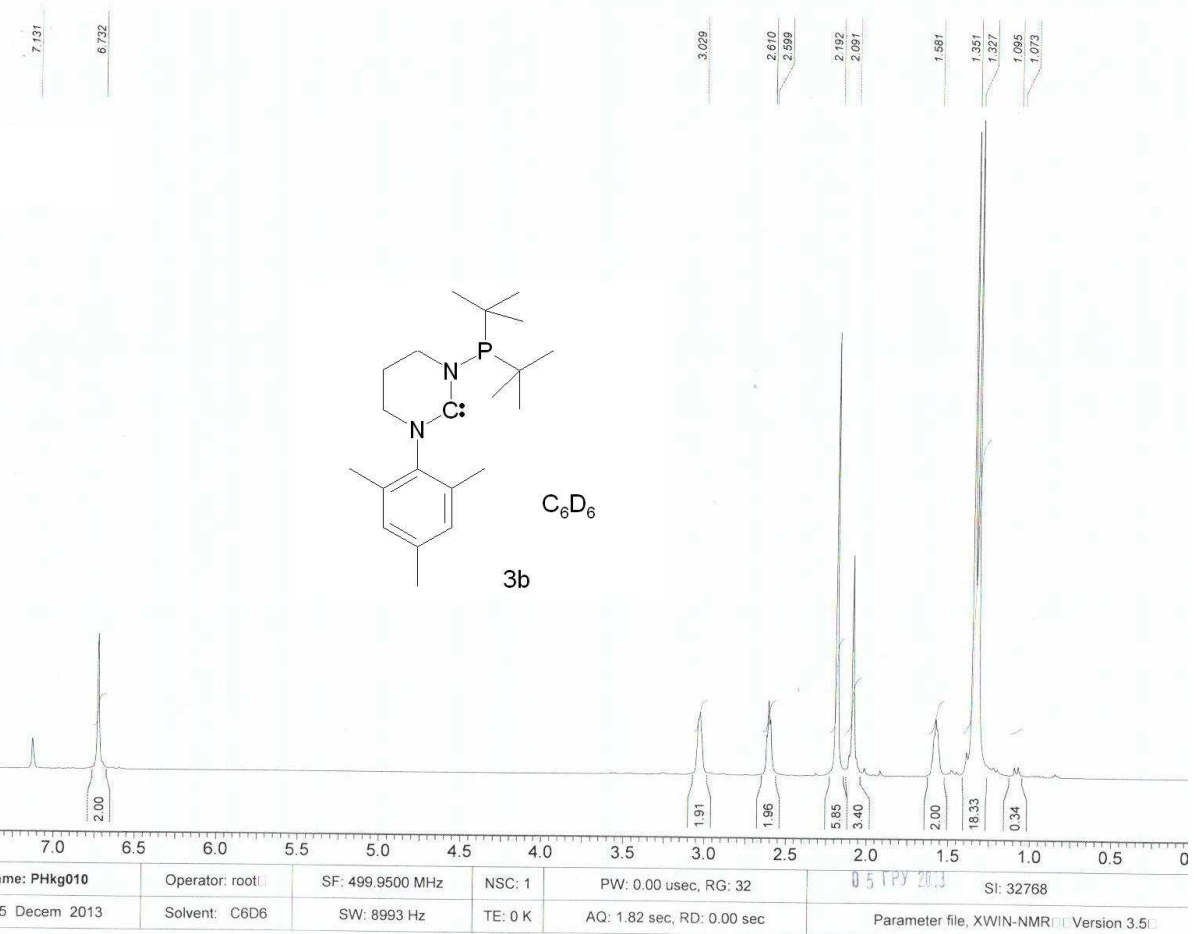

Figure S10. ${ }^{13} \mathrm{C}$ NMR spectrum of $\mathbf{3 b}$

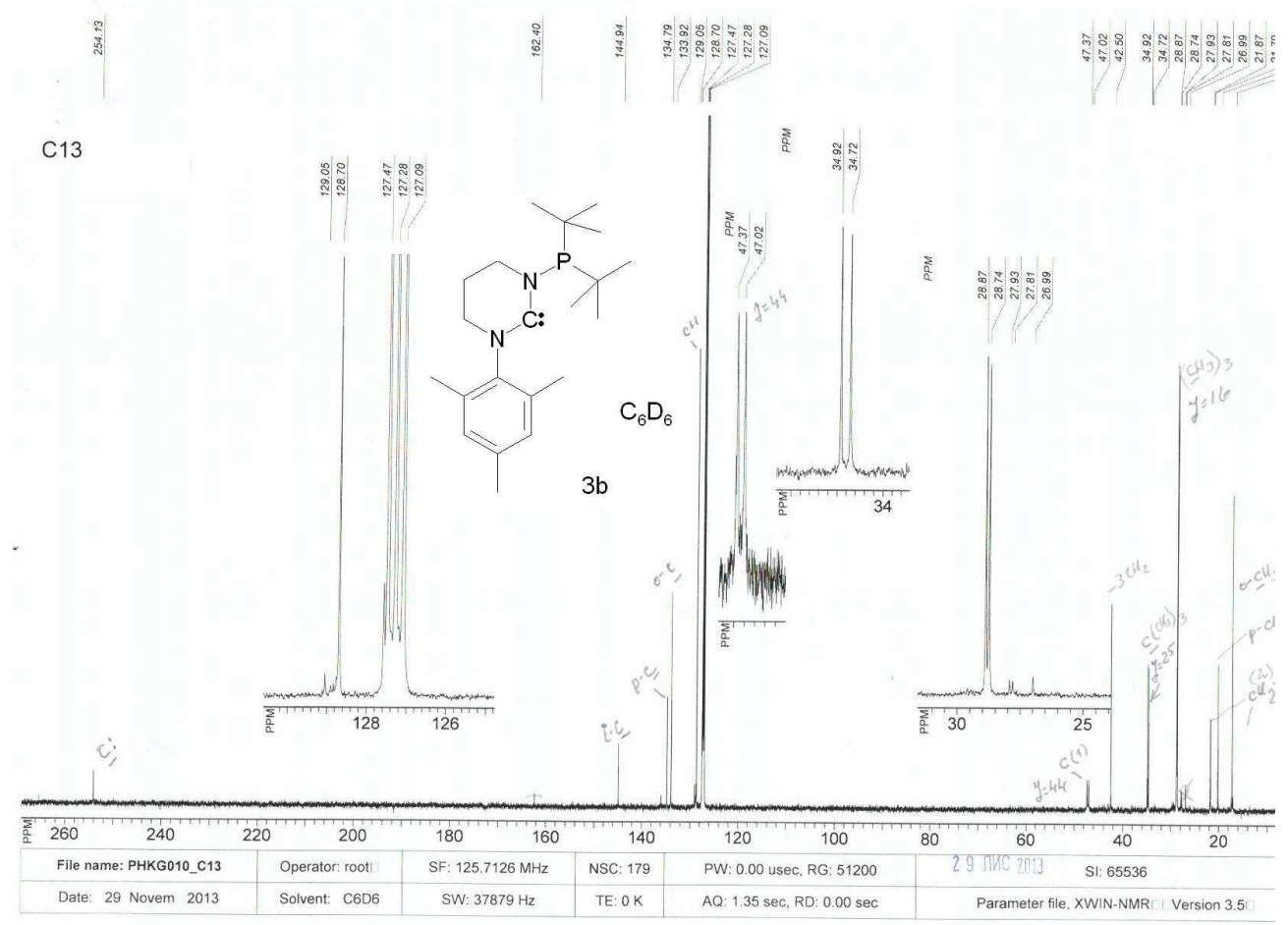


Figure S11. ${ }^{1} \mathrm{H}$ NMR spectrum of $\mathbf{4 a}$

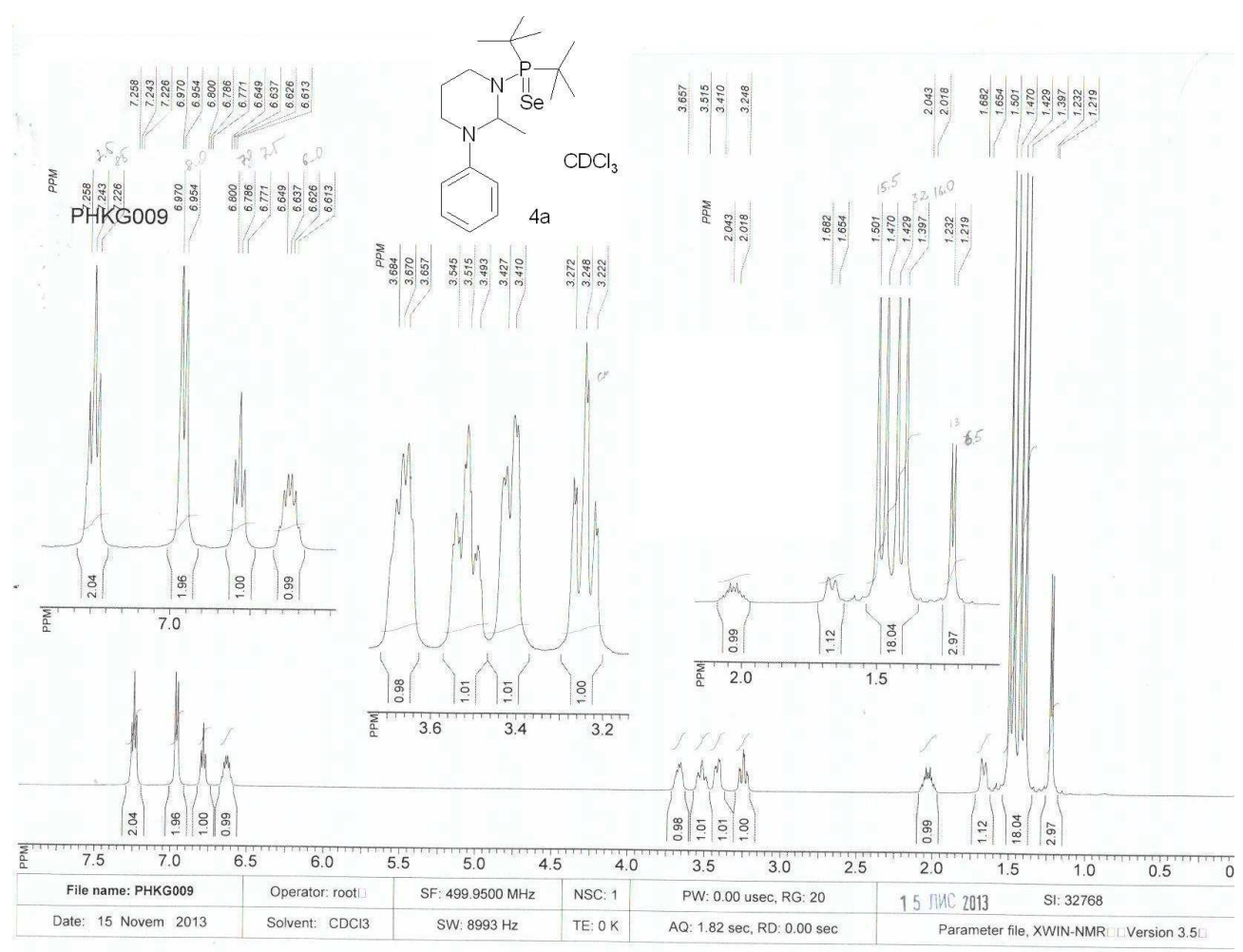

Figure S12. ${ }^{13} \mathrm{C}$ NMR spectrum of $\mathbf{4 a}$

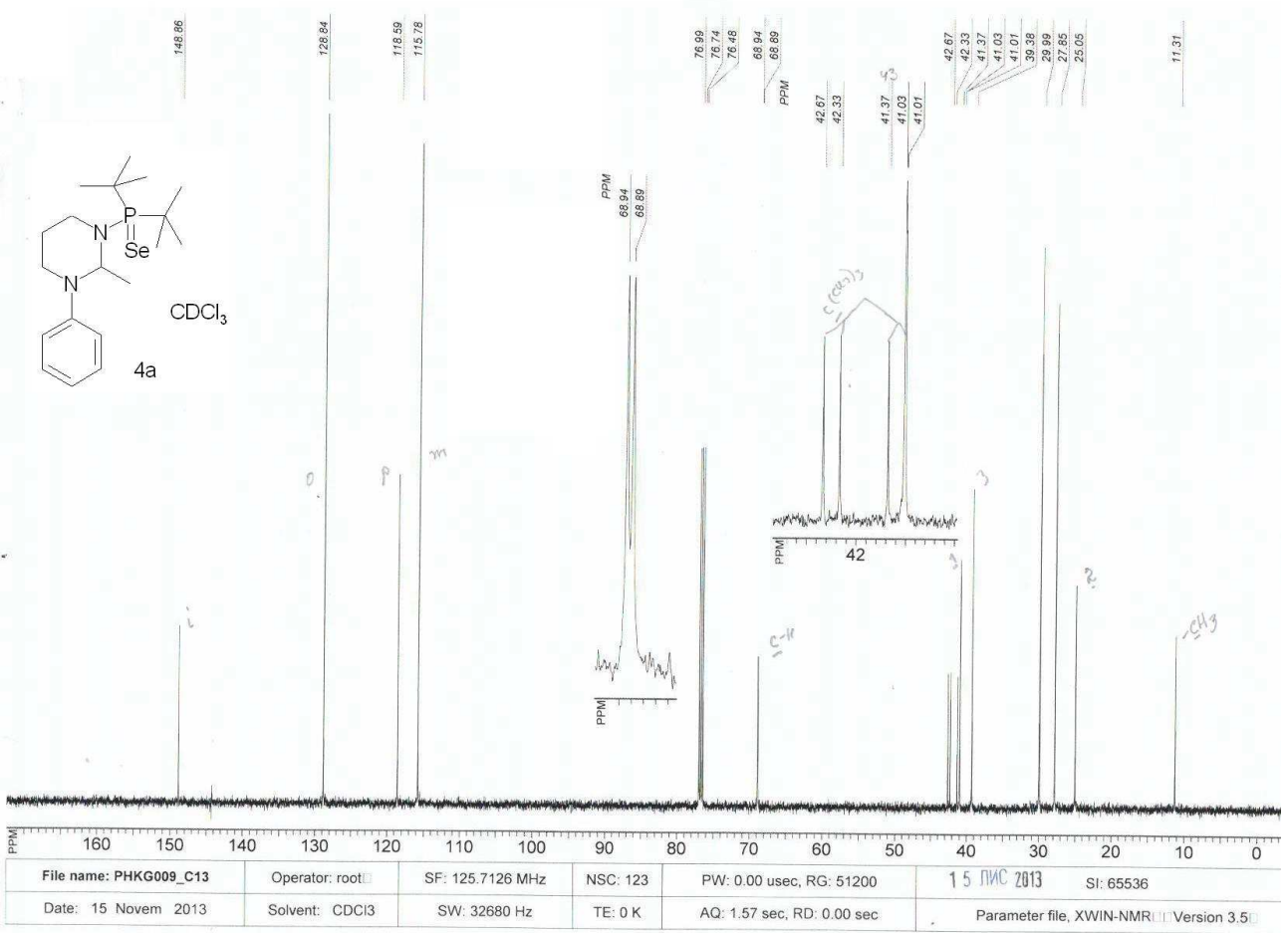


Figure S13. ${ }^{1} \mathrm{H}$ NMR spectrum of $\mathbf{4 b}$

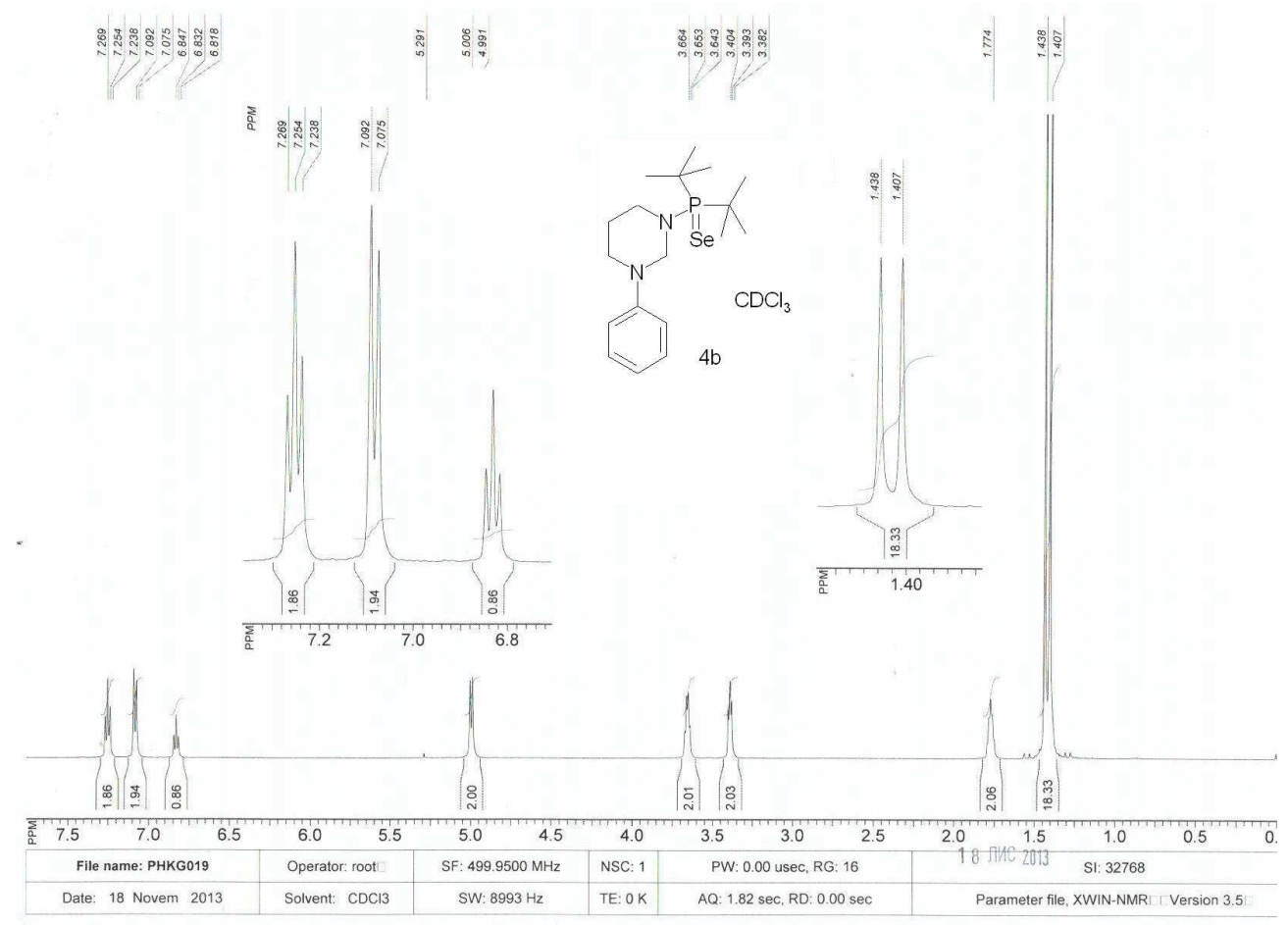

Figure S14. ${ }^{13} \mathrm{C}$ NMR spectrum of $\mathbf{4 b}$

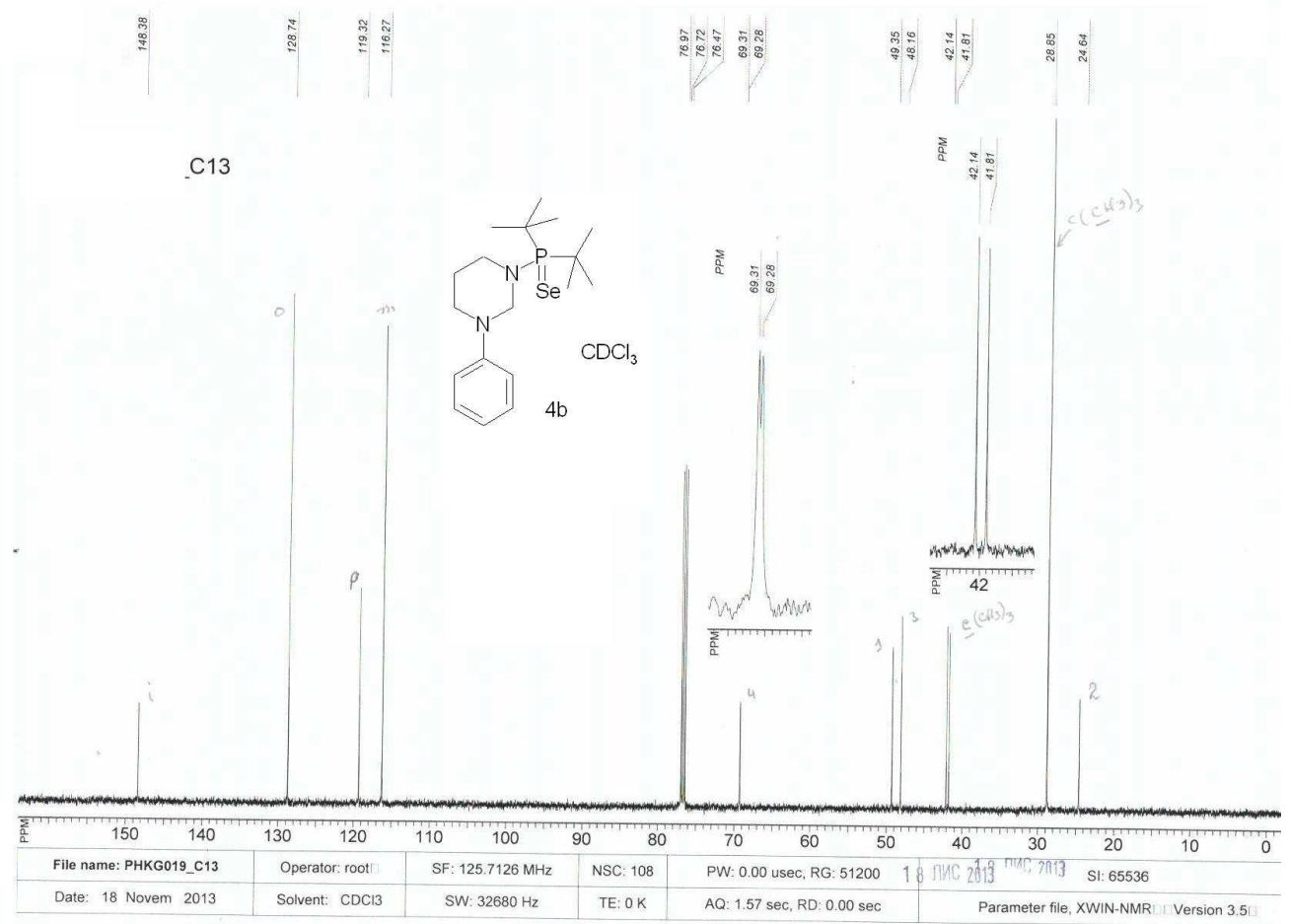


Figure S15. ${ }^{1} \mathrm{H}$ NMR spectrum of $\mathbf{5 a}$

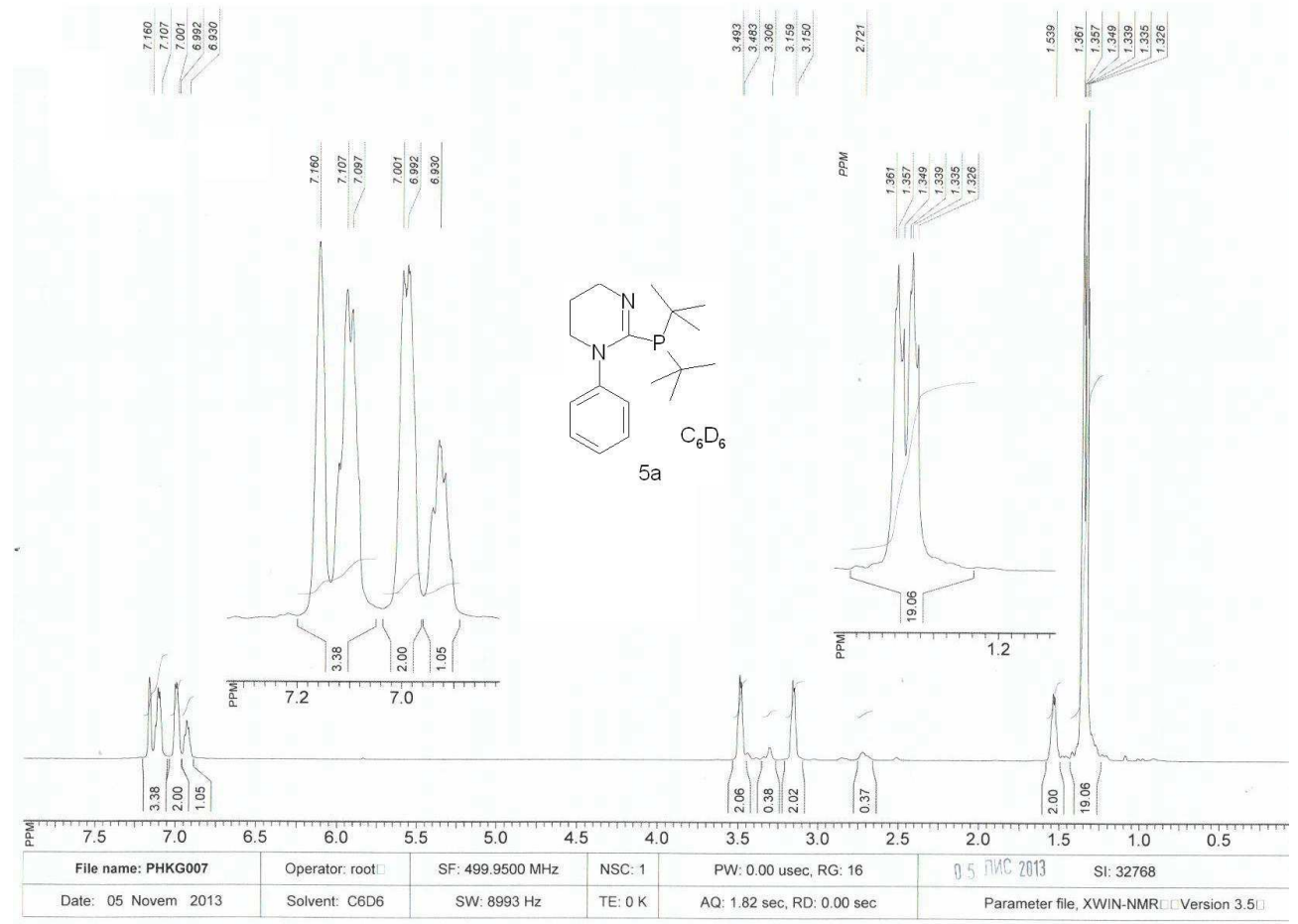

Figure S16. ${ }^{13} \mathrm{C}$ NMR spectrum of $\mathbf{5 a}$

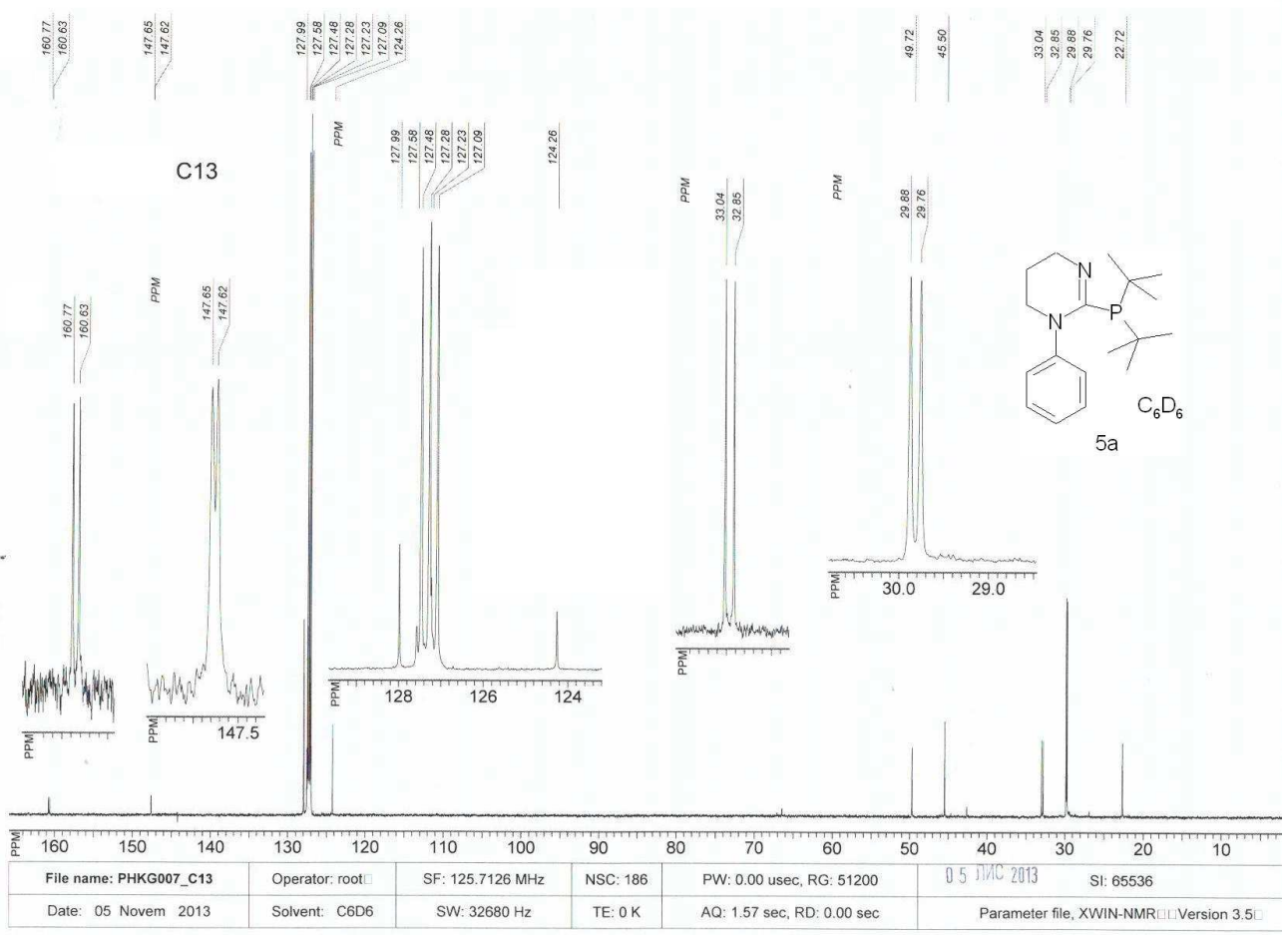


Figure S17. ${ }^{1} \mathrm{H}$ NMR spectrum of $\mathbf{5 b}$

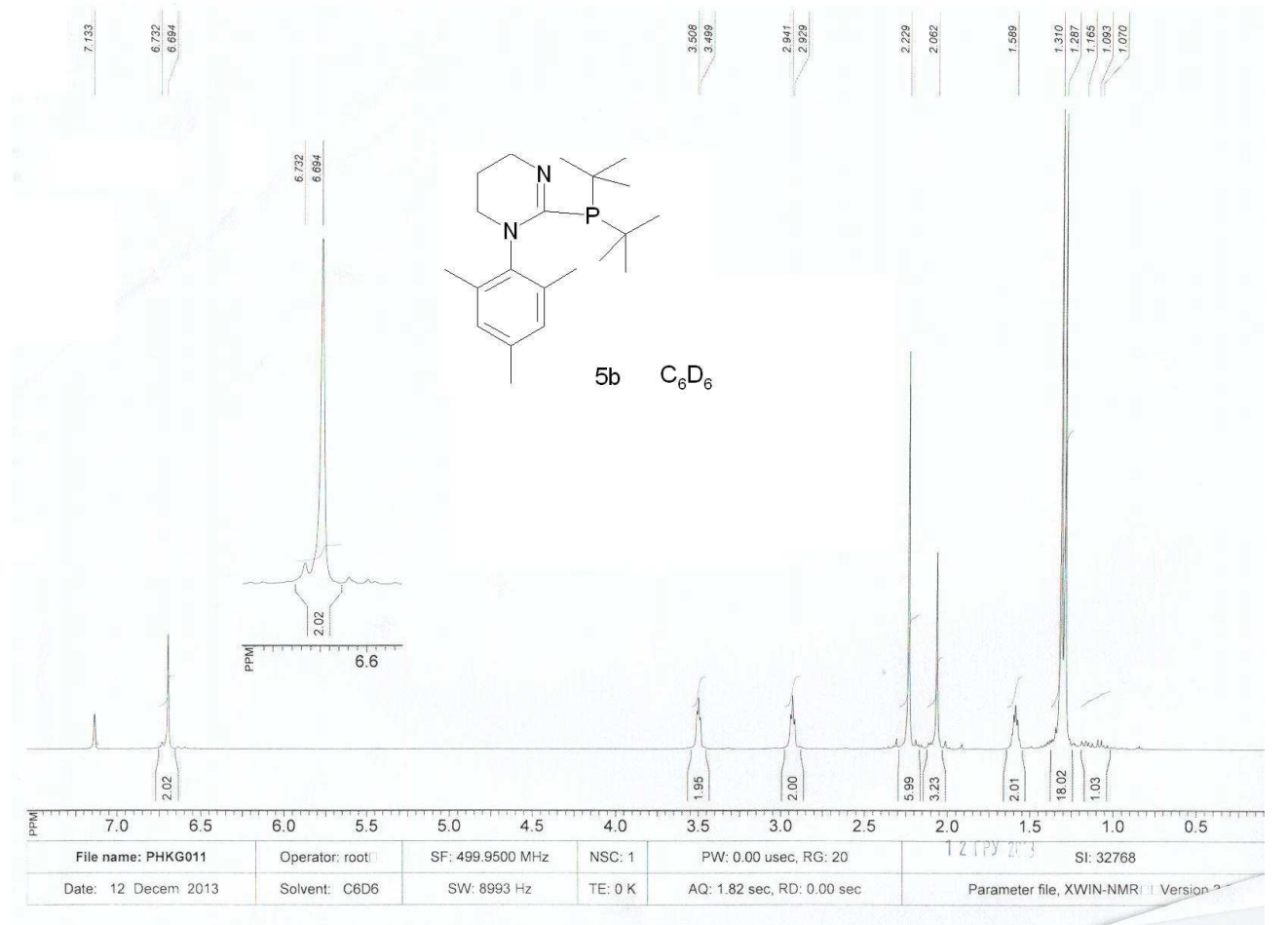

Figure S18. ${ }^{13} \mathrm{C}$ NMR spectrum of $\mathbf{5 b}$

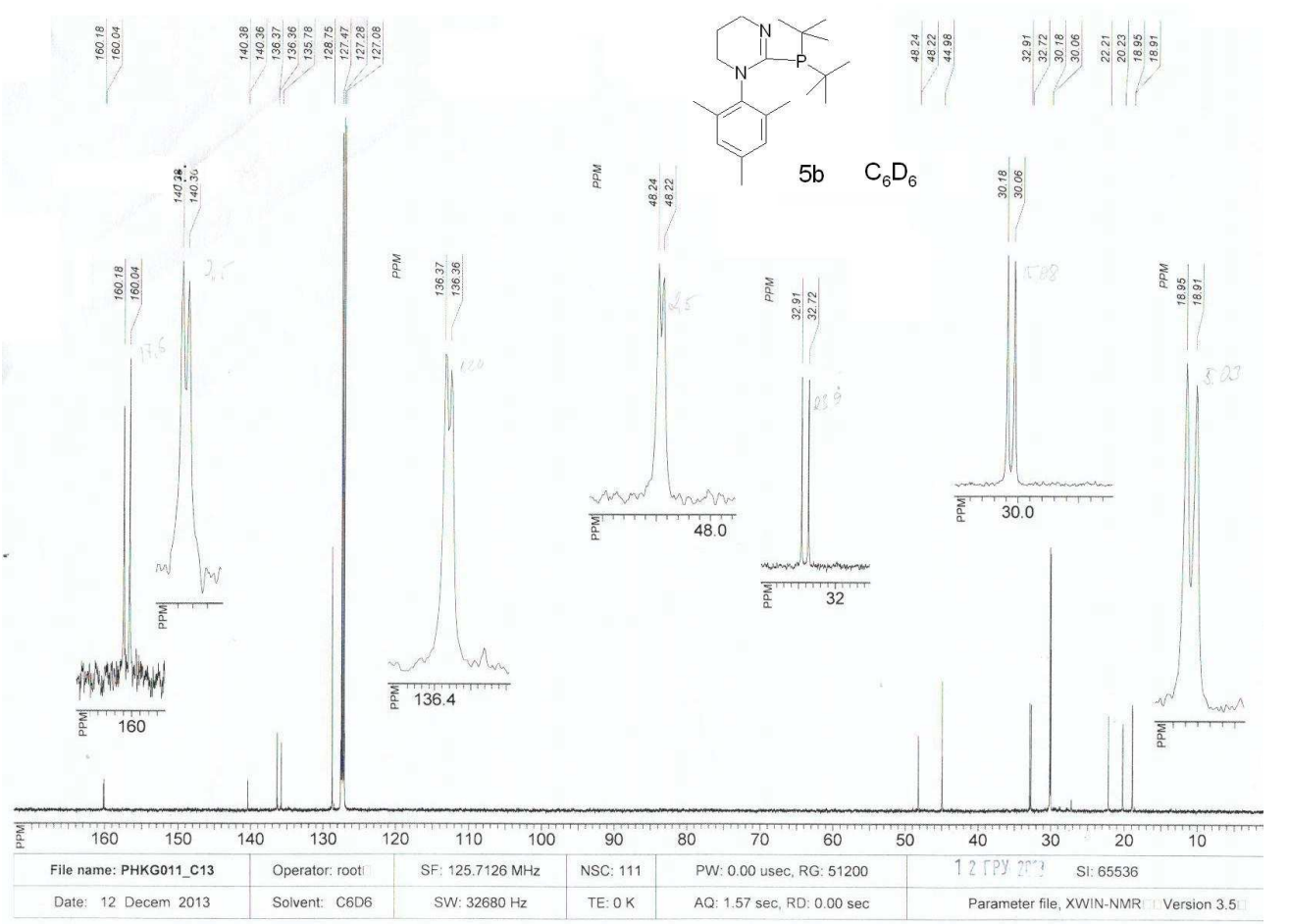


Figure S19. ${ }^{1} \mathrm{H}$ NMR spectrum of $\mathbf{6 a}$

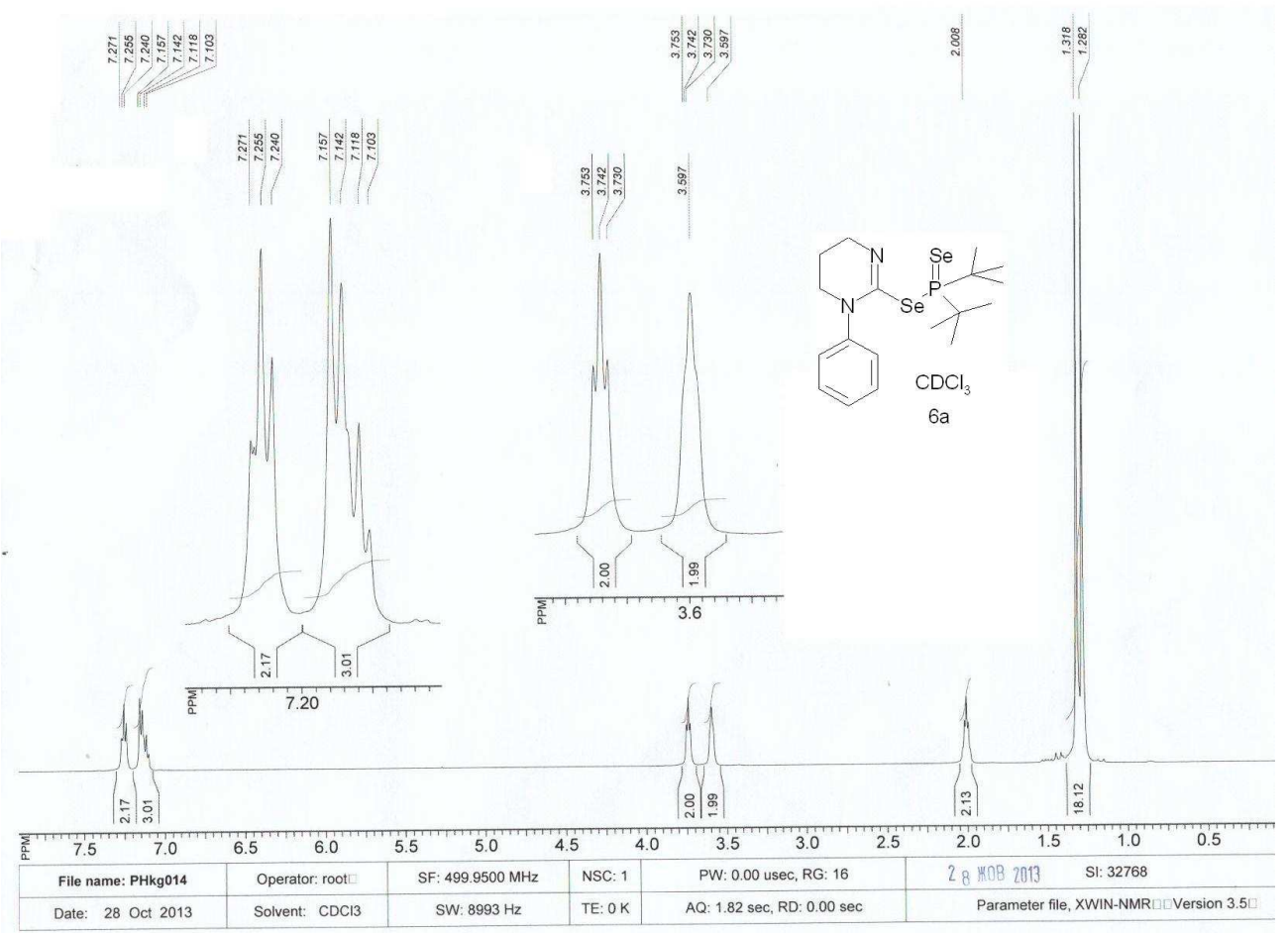

Figure S20. ${ }^{13} \mathrm{C}$ NMR spectrum of $\mathbf{6 a}$

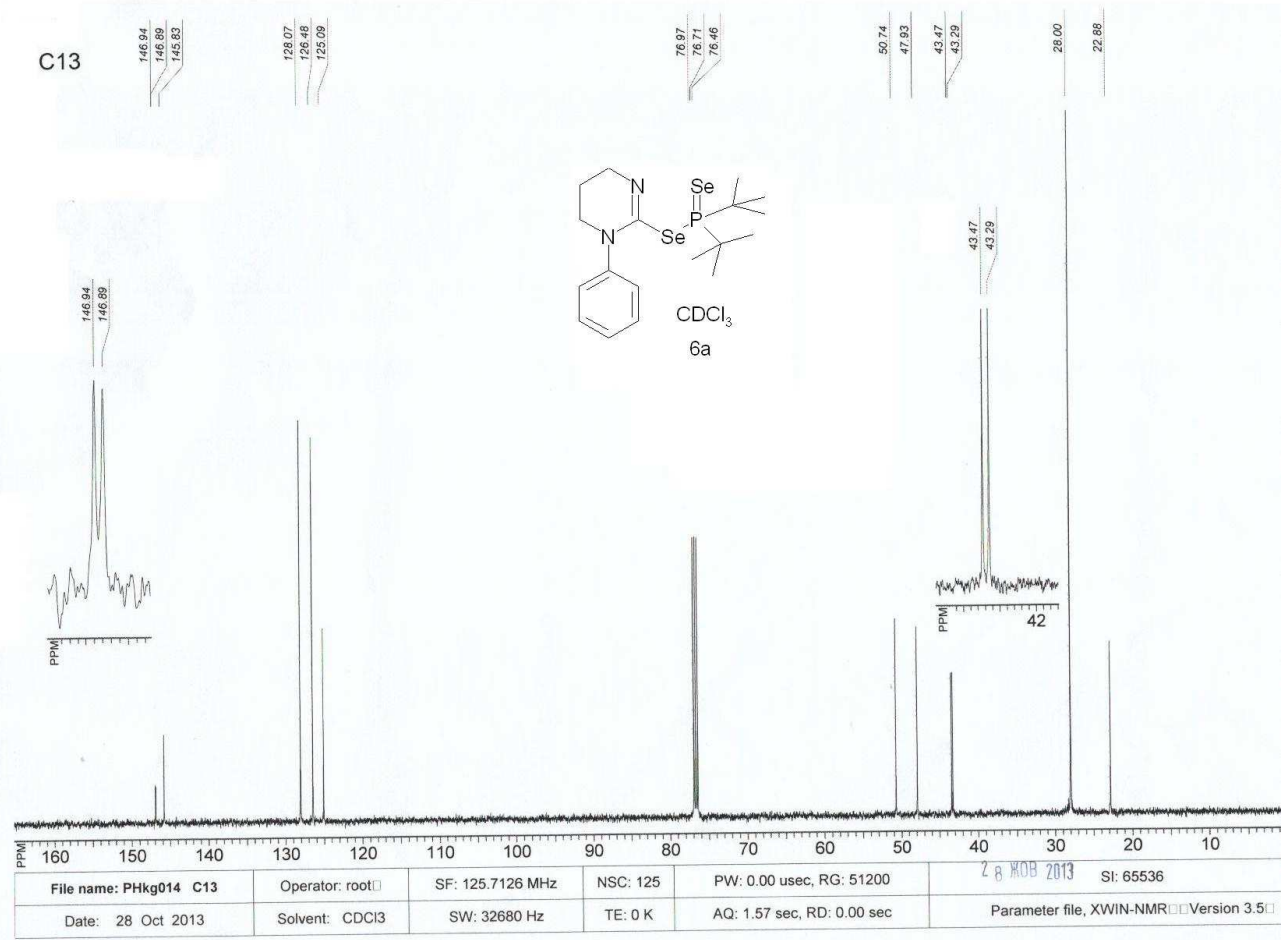


Figure S21. ${ }^{31} \mathrm{P}$ NMR spectrum of $\mathbf{6 a}$

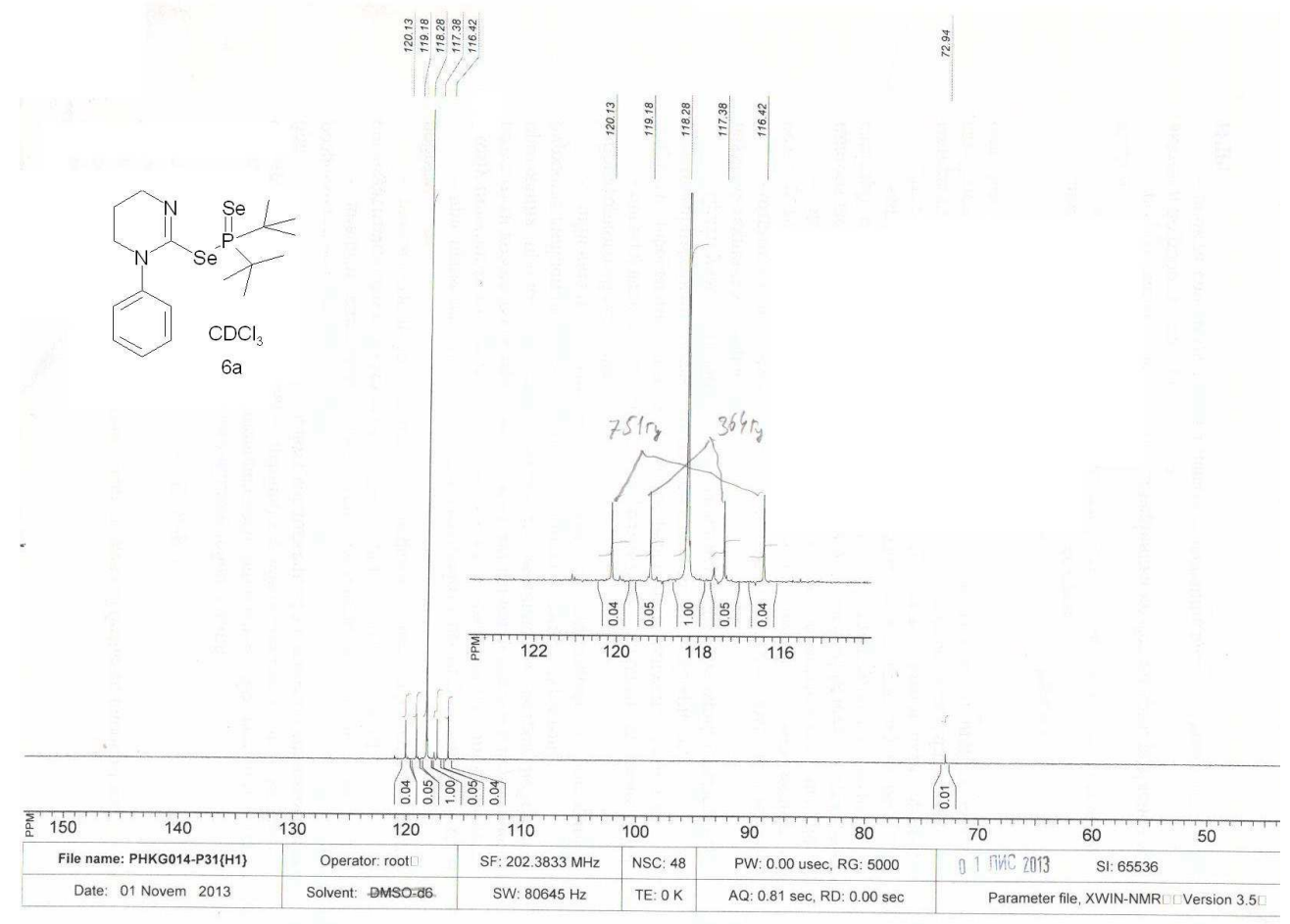

Figure S22. ${ }^{1} \mathrm{H}$ NMR spectrum of $\mathbf{6 b}$

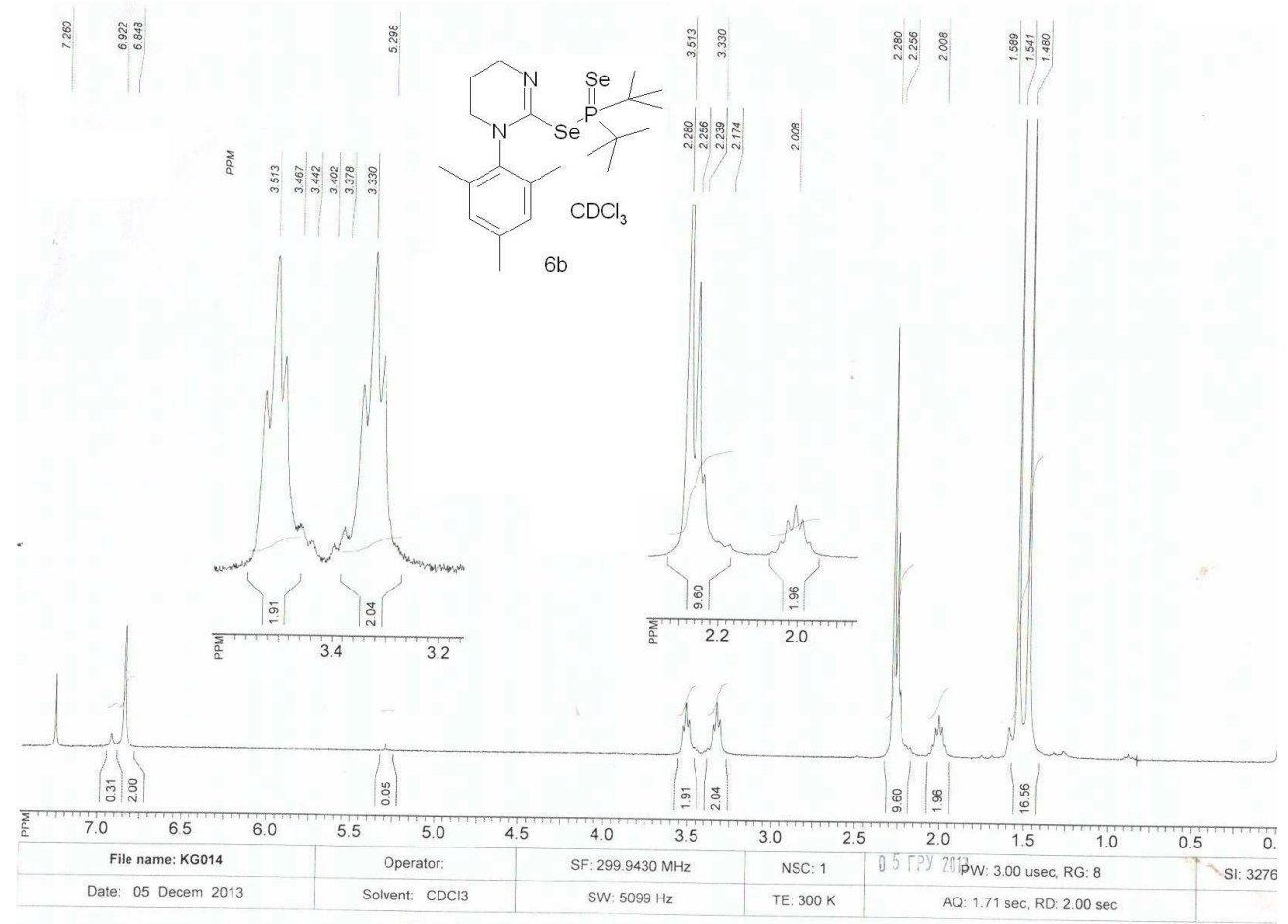


Figure S23. ${ }^{13} \mathrm{C}$ NMR spectrum of $\mathbf{6 b}$

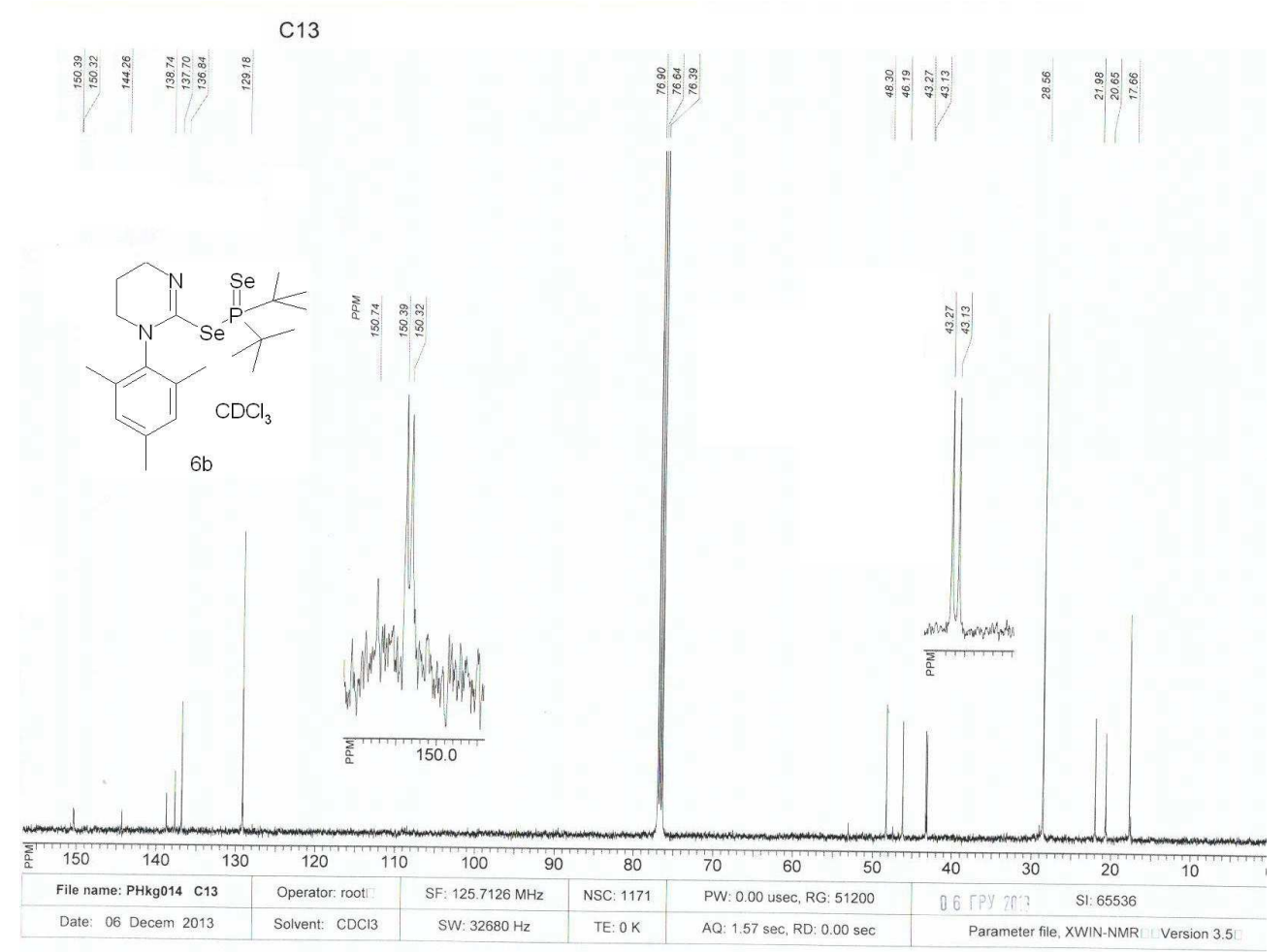

Figure S24. ${ }^{31} \mathrm{P}$ NMR spectrum of $\mathbf{6 b}$

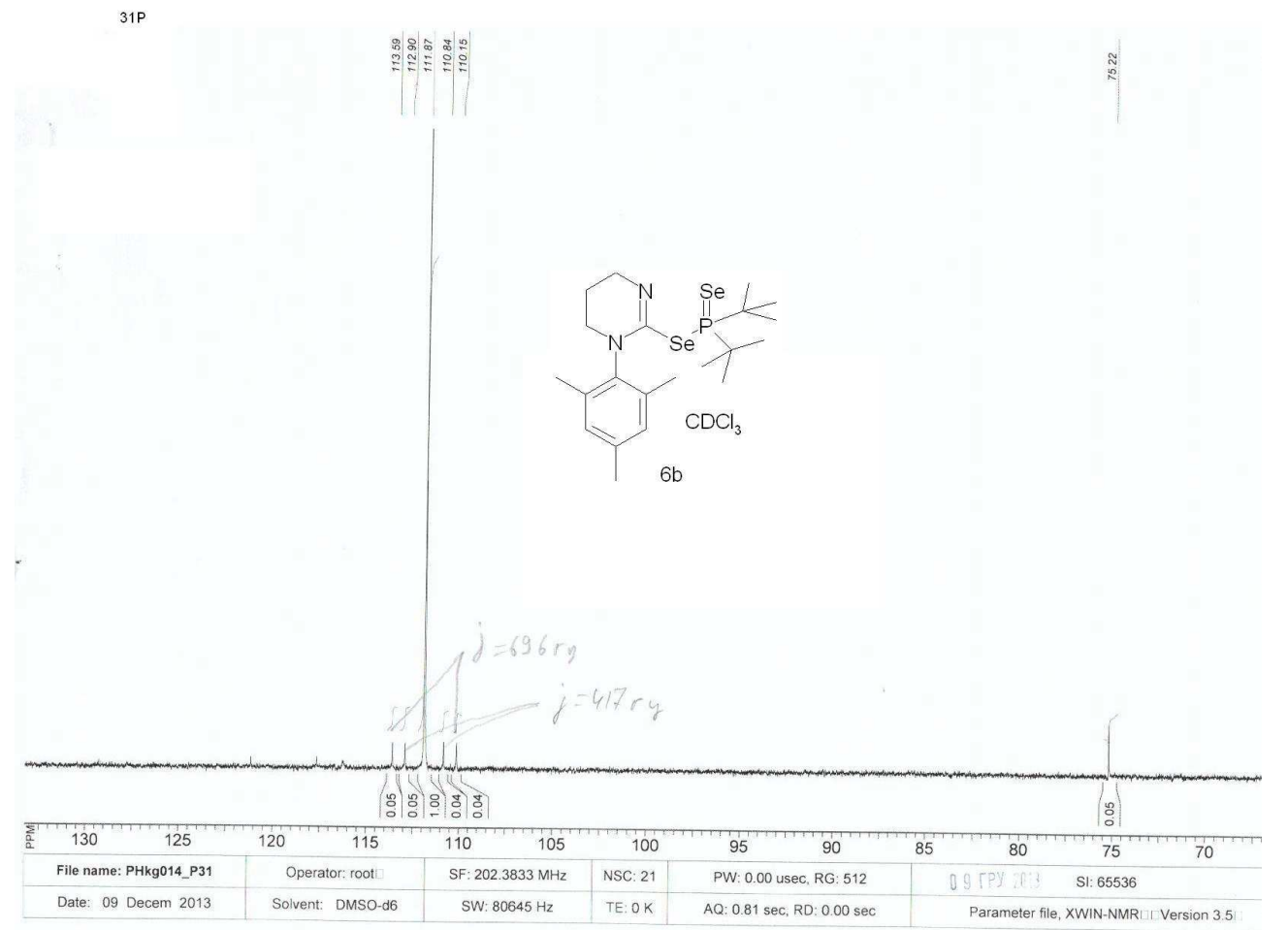


Figure S25. ${ }^{1} \mathrm{H}$ NMR spectrum of $\mathbf{7 a}$

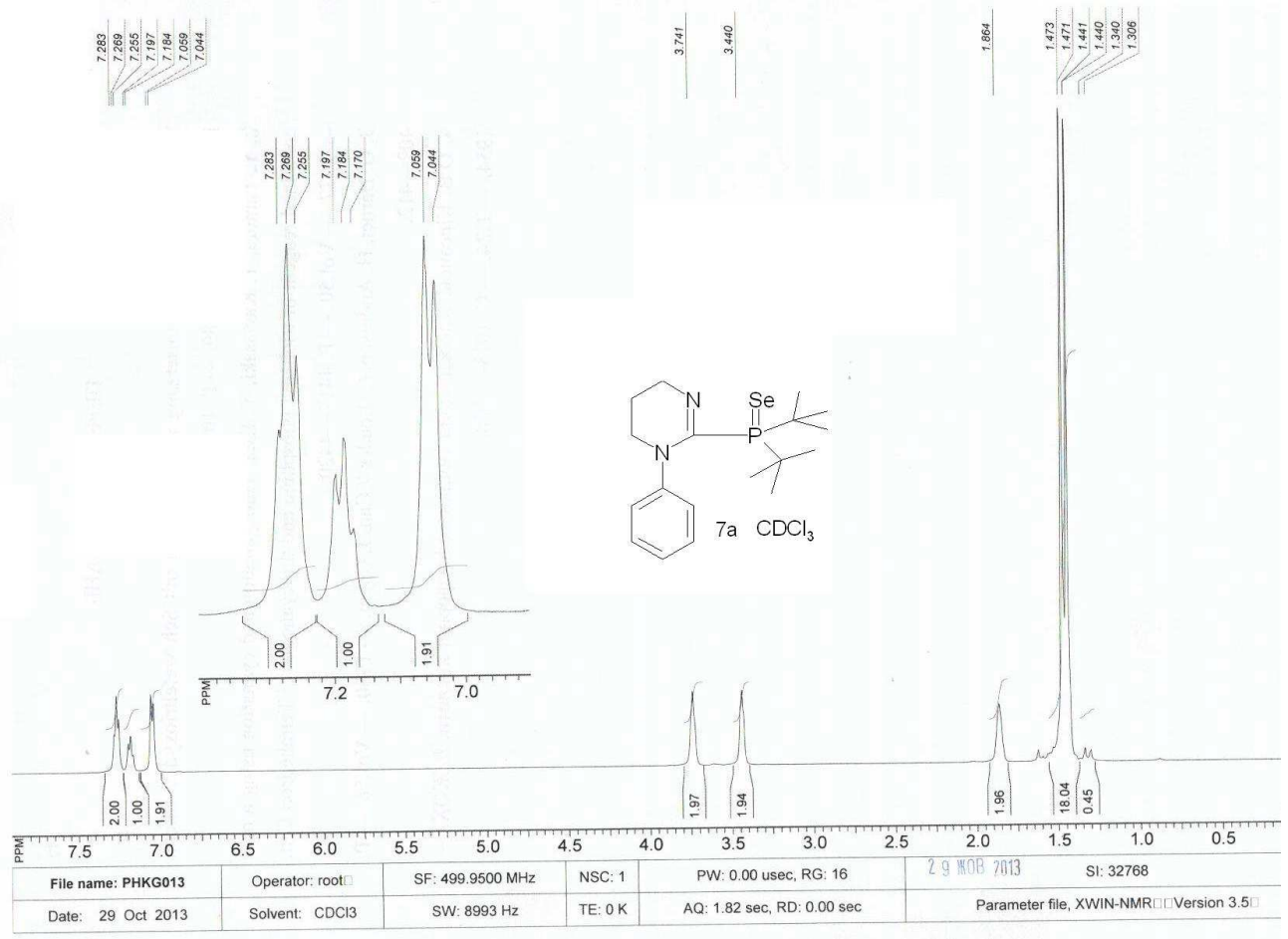

Figure S26. ${ }^{13} \mathrm{C}$ NMR spectrum of $7 \mathbf{a}$

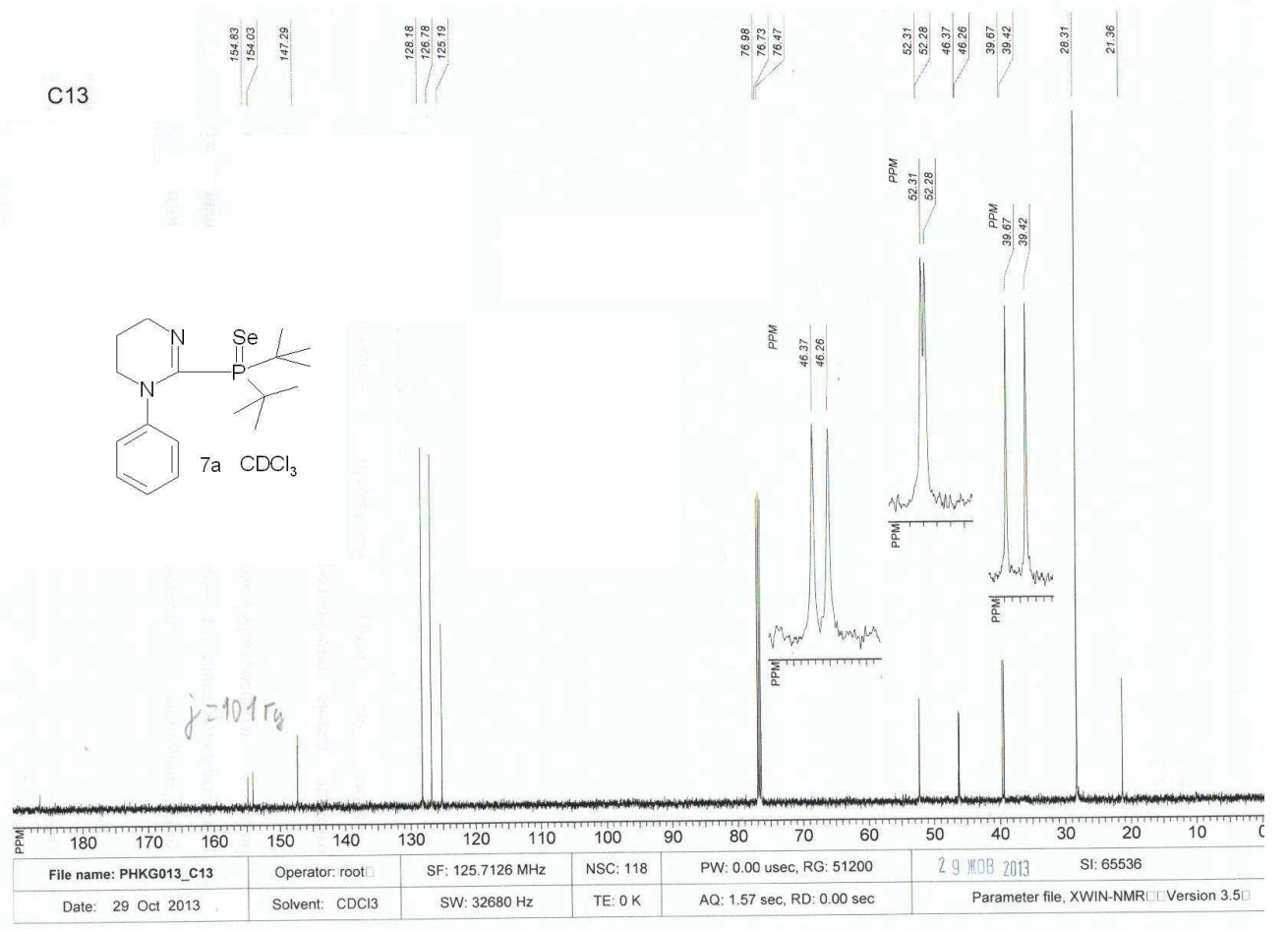


Figure S27. ${ }^{31} \mathrm{P}$ NMR spectrum of $7 \mathbf{a}$

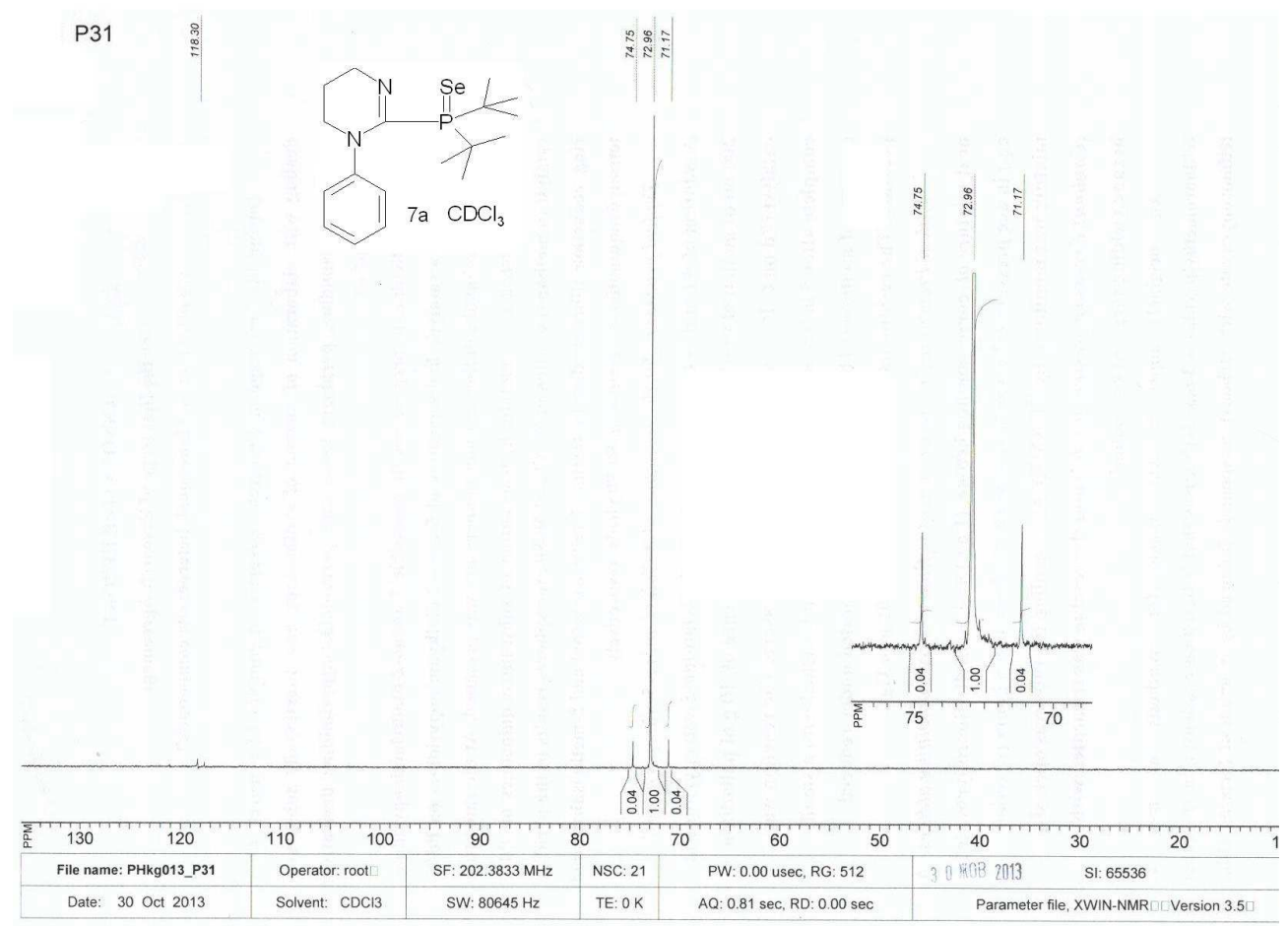

Figure S28. ${ }^{1} \mathrm{H}$ NMR spectrum of $\mathbf{7 b}$

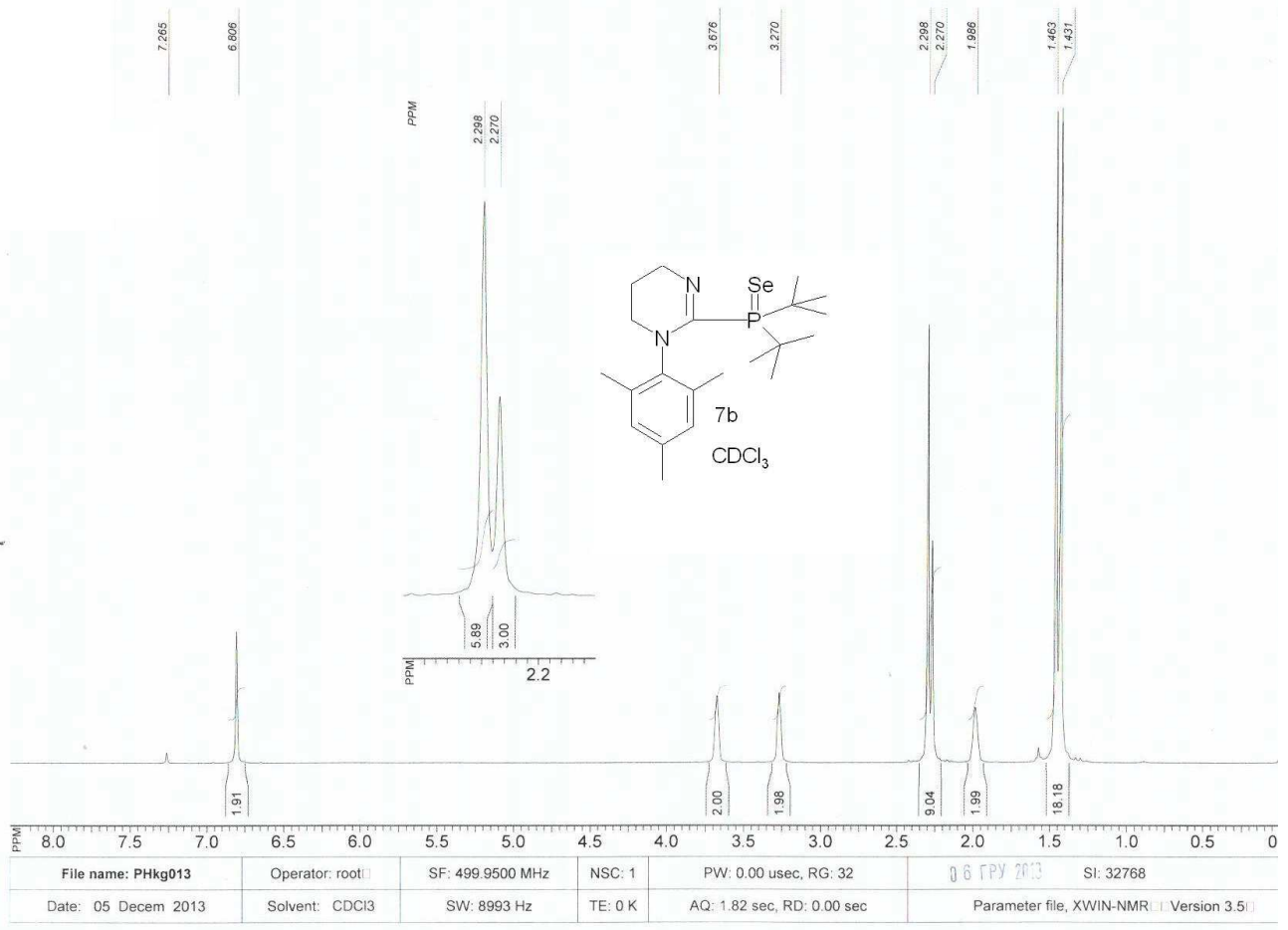


Figure S29. ${ }^{13}$ C NMR spectrum of $\mathbf{7 b}$

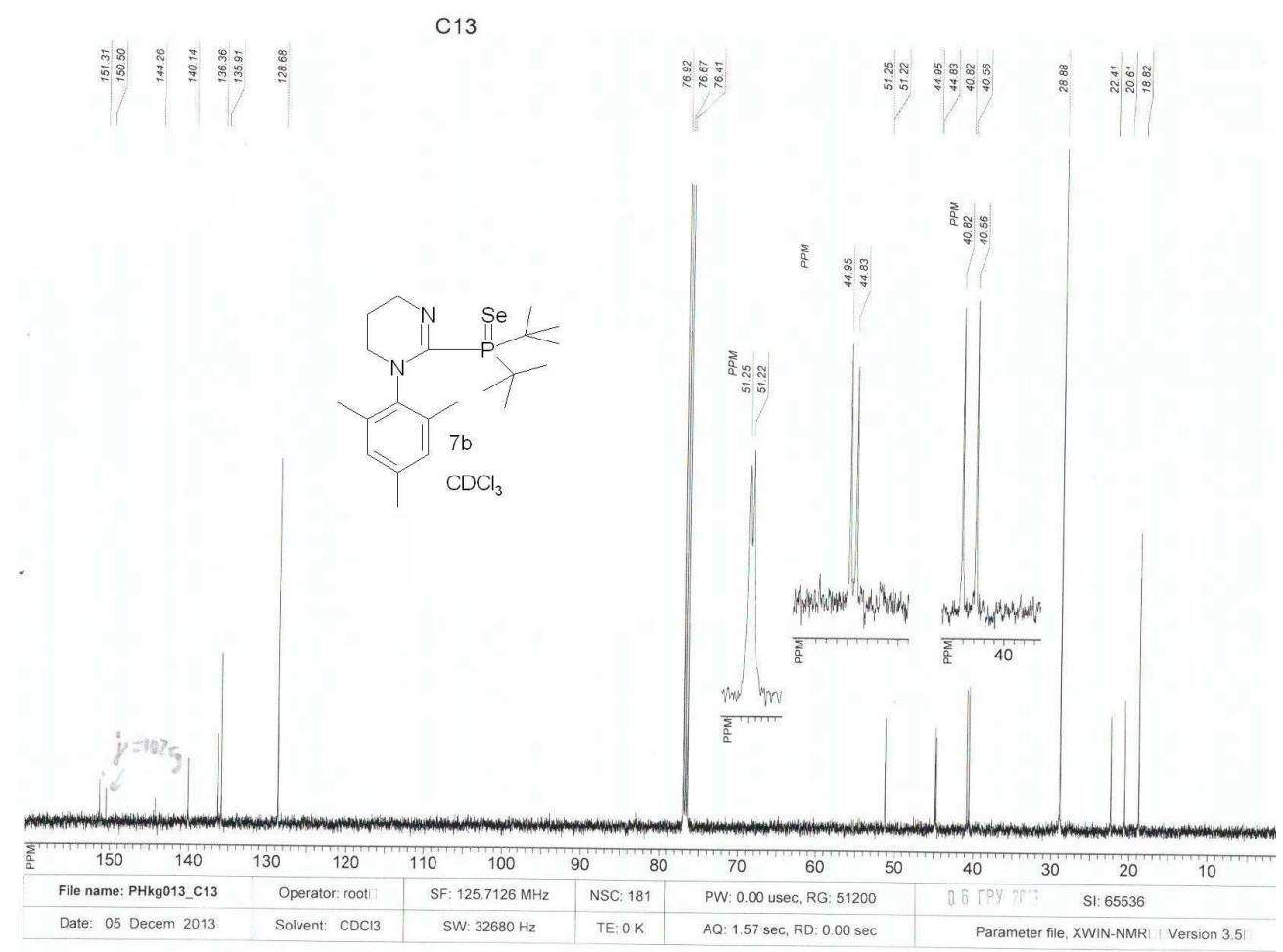

Figure S30. ${ }^{31} \mathrm{P}$ NMR spectrum of $\mathbf{7 b}$

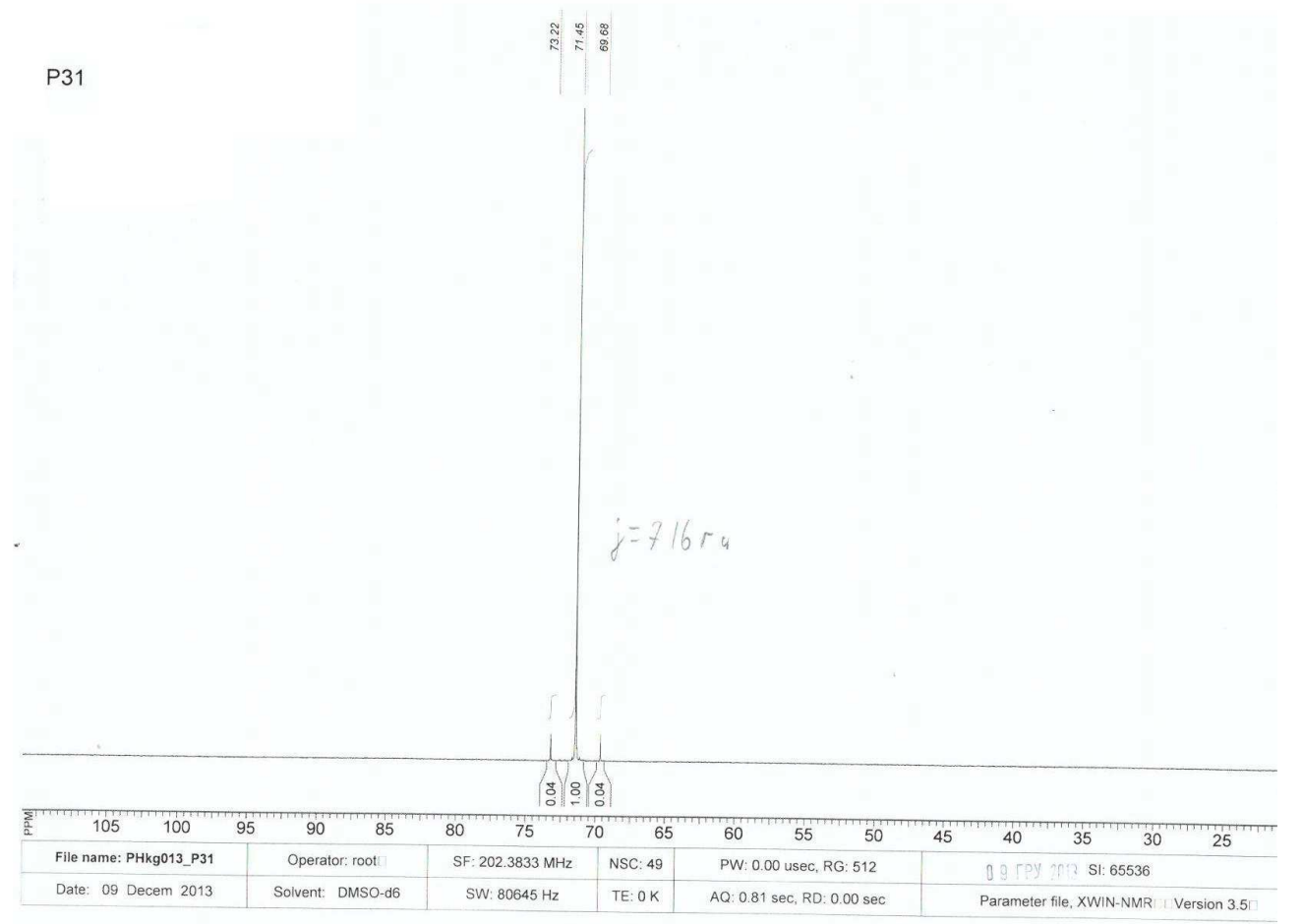


Figure S31. ${ }^{1}$ H NMR spectrum of 8

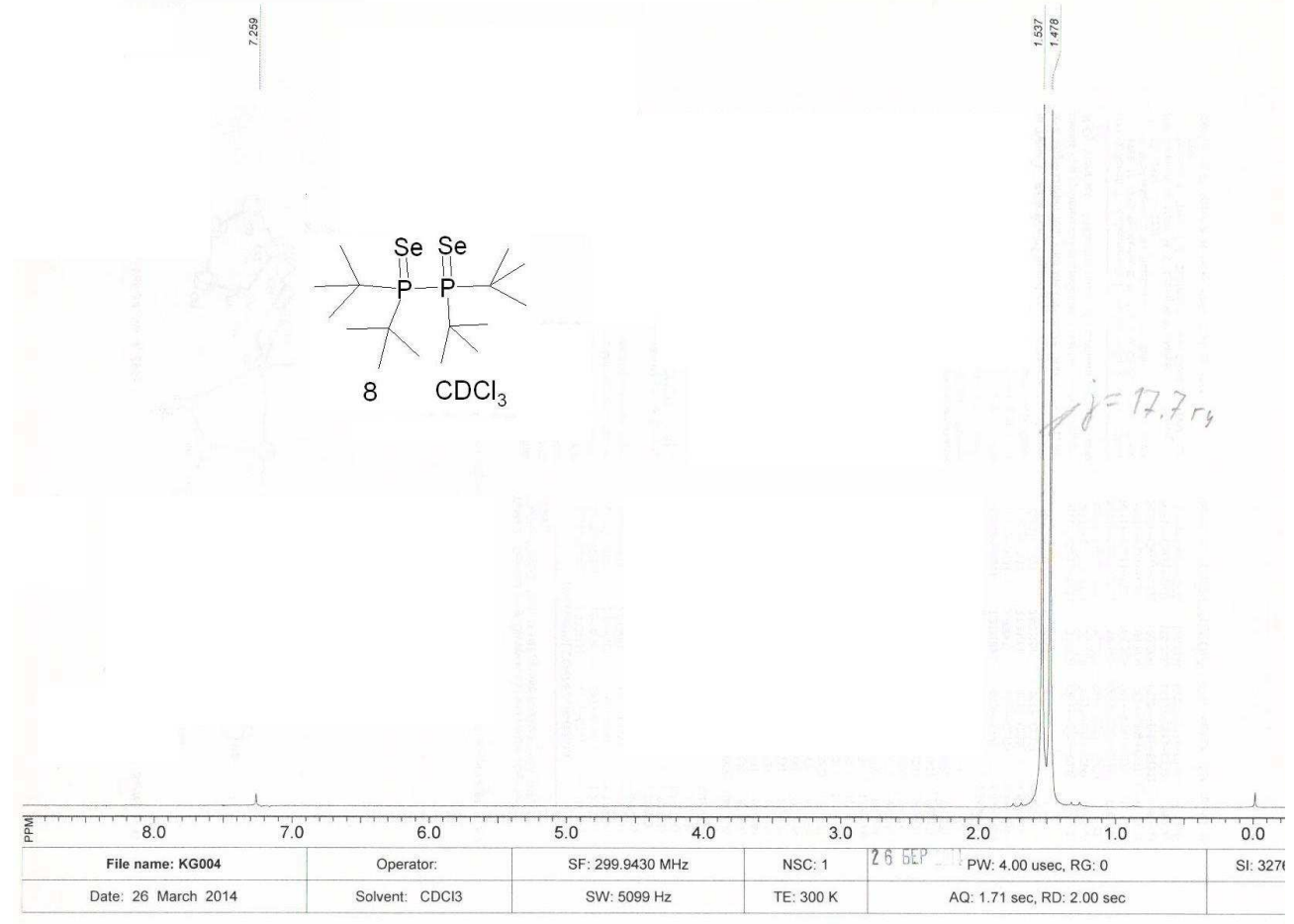

Figure S32. ${ }^{13} \mathrm{C}$ NMR spectrum of 8

C13

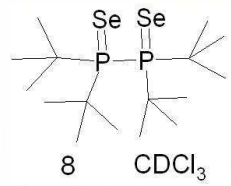

\begin{tabular}{|c|c|c|c|c|c|c|c|}
\hline 这 240 & 200 & 160 & 120 & 100 & $60 \quad 40$ & 20 & 0 \\
\hline File name: PHkg004 C13 & Operator; root & SF: $125,7126 \mathrm{MHz}$ & NSC: 139 & PW: 0.00 usec, RG: 51200 & $255 E P 2914$ & SI: 65536 & \\
\hline Date: 26 March 2014 & Solvent: $\mathrm{CDC} 13$ & SW: $32680 \mathrm{~Hz}$ & TE: OK & $A Q: 1.57 \mathrm{sec}, \mathrm{RD}: 0.00 \mathrm{sec}$ & Parameter file, & e, XWIN-NMR & Version 3.5 \\
\hline
\end{tabular}


Figure S33. ${ }^{31} \mathrm{P}$ NMR spectrum of 8

P31

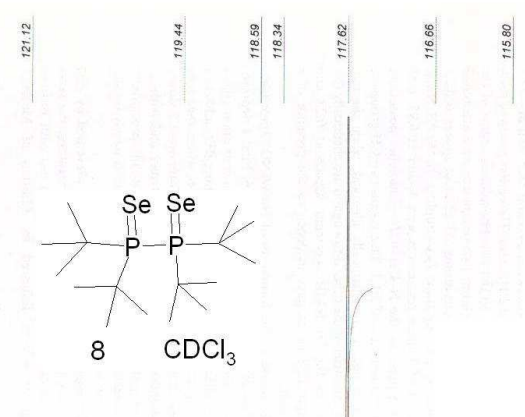

Figure S34. ${ }^{1} \mathrm{H}$ NMR spectrum of $\mathbf{9 a}$

ถำำ:
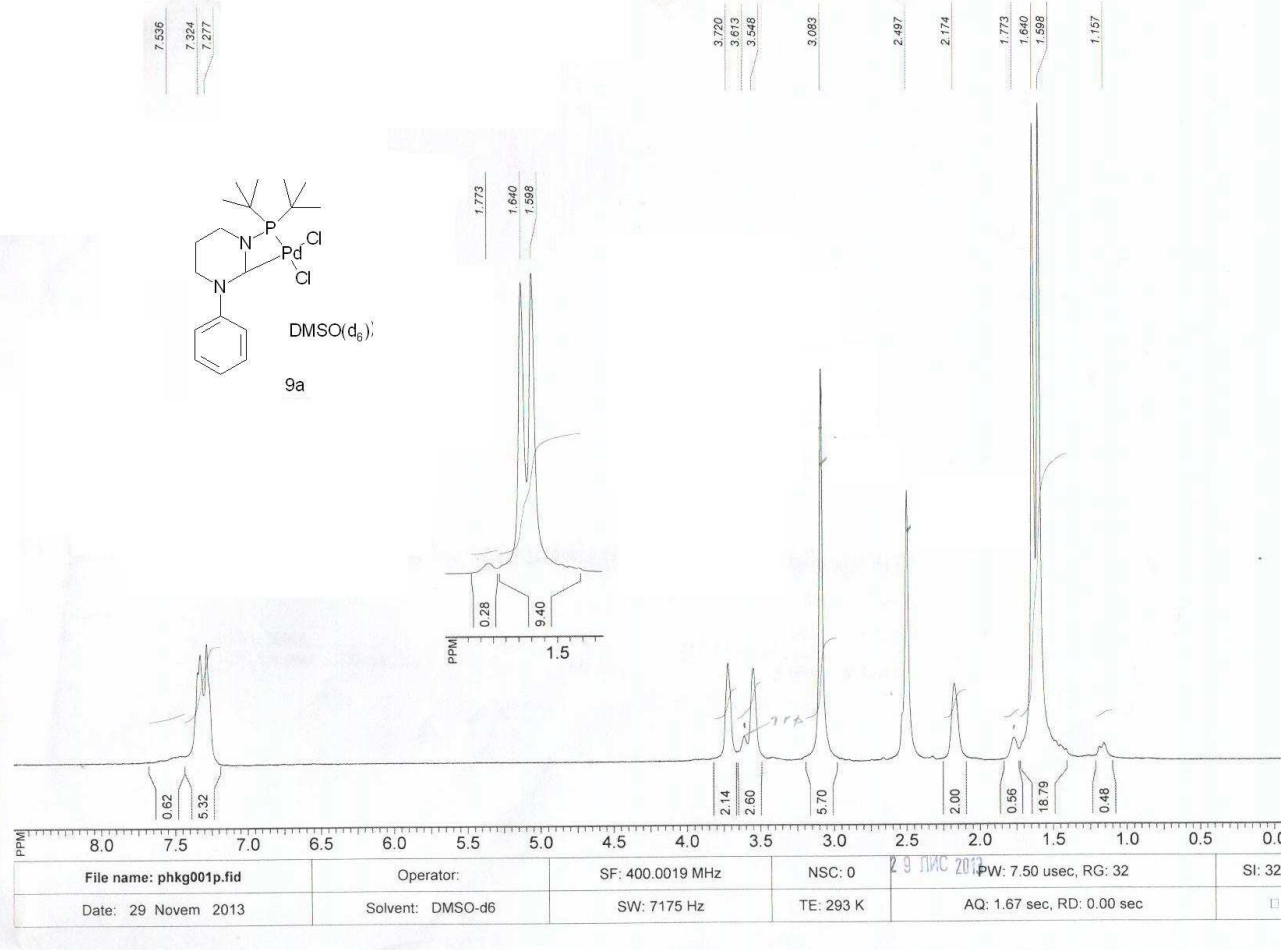
Figure S35. ${ }^{13} \mathrm{C}$ NMR spectrum of $9 a$

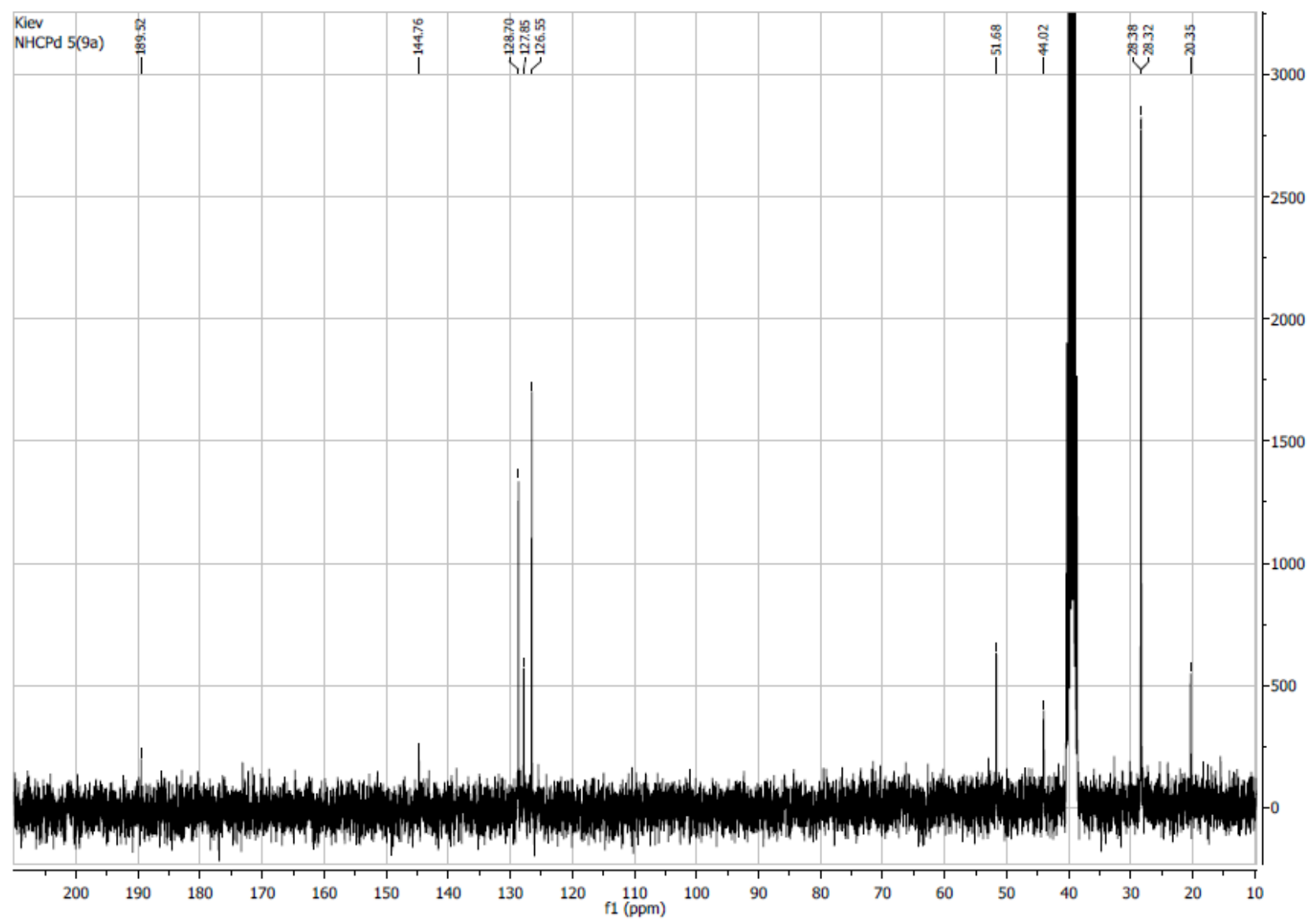

Figure S36. ${ }^{1} \mathrm{H}$ NMR spectrum of $9 \mathbf{b}$

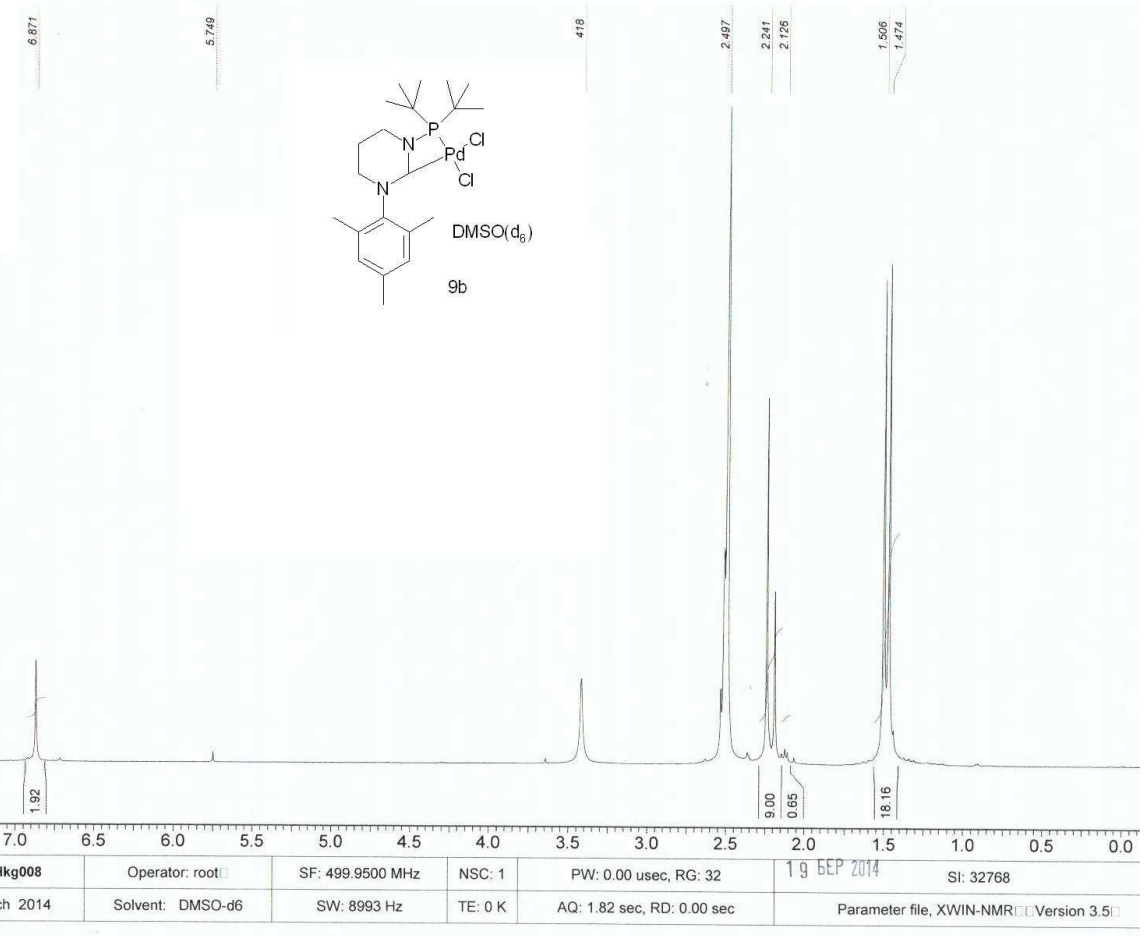

S21 
Figure S37. ${ }^{13} \mathrm{C}$ NMR spectrum of $\mathbf{9 b}$

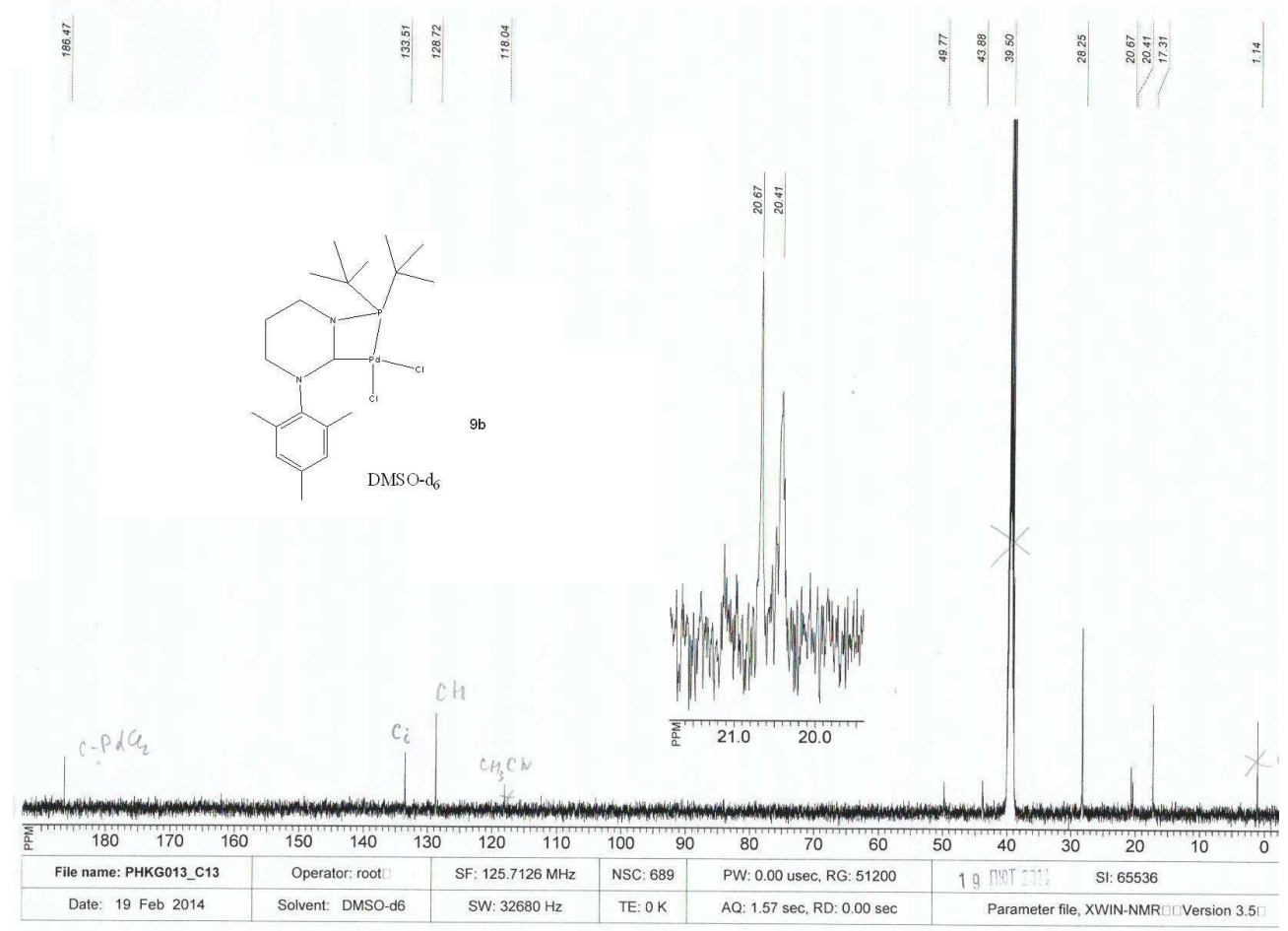

Figure S38. ${ }^{1} \mathrm{H}$ NMR spectrum of $\mathbf{9 c}$

气ิ
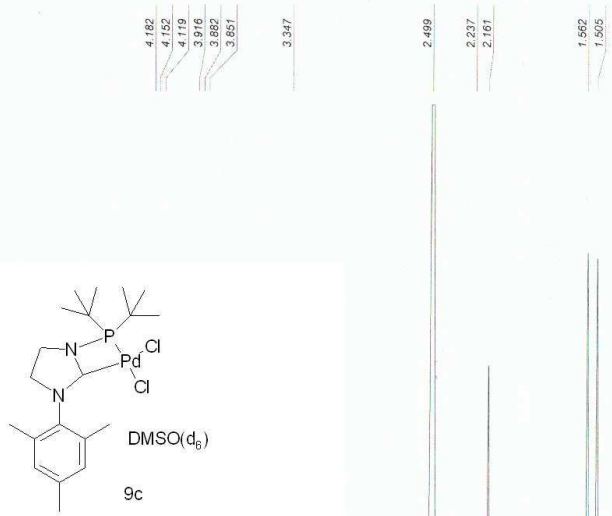

$\frac{2}{2}$

\begin{tabular}{|c|c|c|c|c|c|c|c|c|c|c|c|c|c|c|}
\hline 7.0 & 6.5 & 6.0 & 5.5 & 5.0 & 4.5 & 4.0 & 3.5 & 3.0 & 2.5 & 2.0 & 1.5 & 1.0 & 0.5 & 0.0 \\
\hline & $\mathrm{kg}$ & & & Operator & & & $9450 \mathrm{MHz}$ & & NSC: 10 & 19. rPy & PW: 4.0 & $c, R G: 0$ & & SI: 32 \\
\hline
\end{tabular}

Date: 19 Decem 2013

Solvent: DMSO-d6

SW: $5099 \mathrm{~Hz}$

TE: $300 \mathrm{~K}$

$A Q: 1.71 \mathrm{sec}, \mathrm{RD}: 2.00 \mathrm{sec}$ 
Figure S39. ${ }^{13} \mathrm{C}$ NMR spectrum of $9 c$

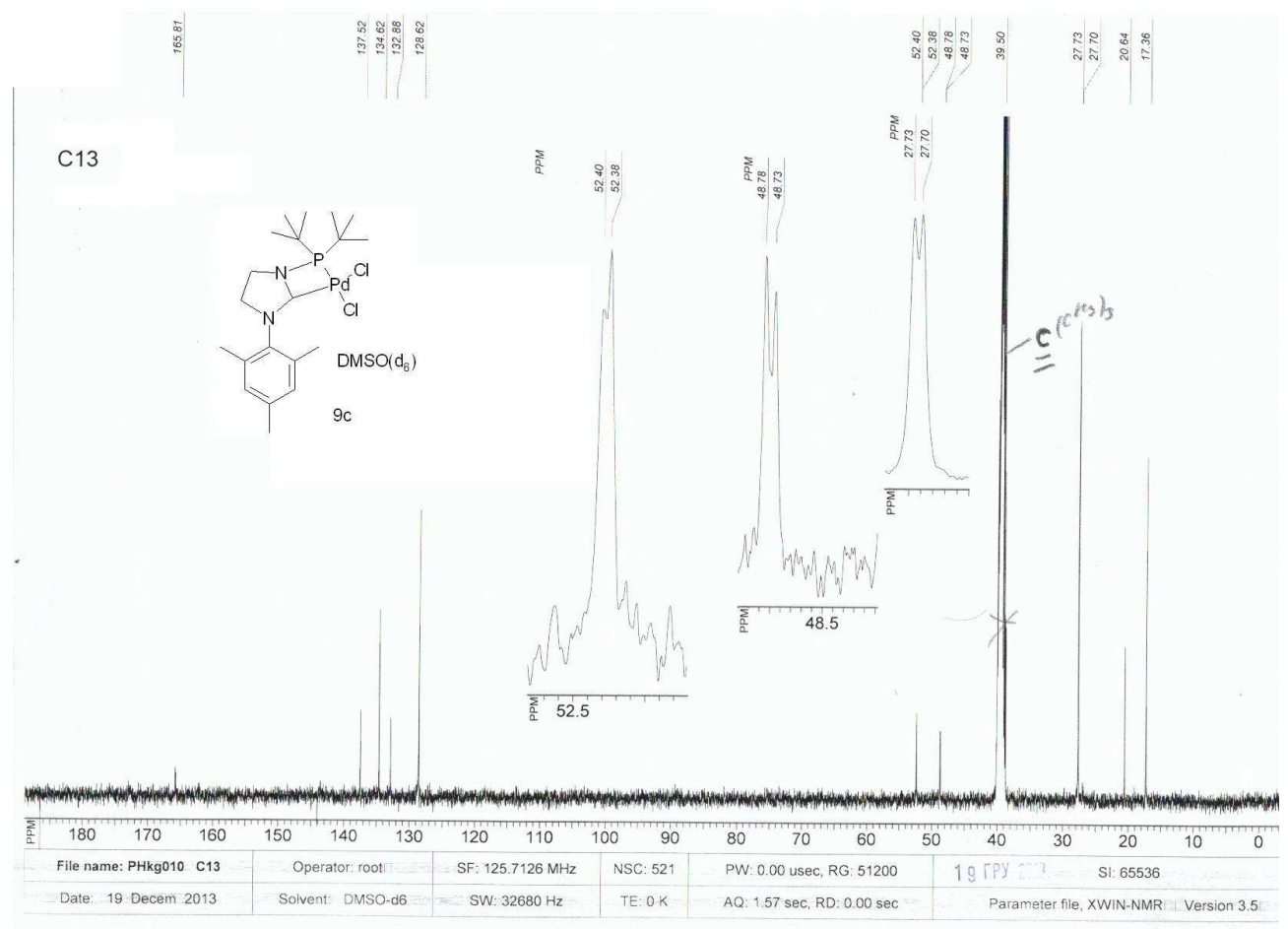

